\title{
DIMENSIONS OF PRODUCTS OF HYPERBOLIC SPACES
}

\author{
N. LEBEDEVA
}

To dear Viktor Abramovich Zalgaller on the occasion of his 85th birthday

\begin{abstract}
Estimates on asymptotic dimension are given for products of general hyperbolic spaces, with applications to hyperbolic groups. Examples are presented where strict inequality occurs in the product theorem for the asymptotic dimension in the class of hyperbolic groups and in the product theorem for the hyperbolic dimension. It is proved that $\mathbb{R}$ is dimensionally full for the asymptotic dimension in the class of hyperbolic groups.
\end{abstract}

\section{$\S 1$. INTRODUCTION}

Our purpose in this paper is to answer some questions concerning the theory of asymptotic dimension (in particular, those posed in [Dr3]). For this, we give various estimates on the asymptotic dimension of general hyperbolic spaces and products of hyperbolic spaces and apply these estimates to hyperbolic groups.

To formulate our results we need some definitions. We say that the metric spaces $\left\{X_{\alpha}: \alpha \in \mathcal{A}\right\}$ have linearly controlled dimension $\ell$-dim not exceeding $k$ uniformly if there is a constant $\delta \in(0,1)$ such that for every sufficiently small $\tau>0$ and every $\alpha \in \mathcal{A}$ there exists a $(k+1)$-colored open covering $\mathcal{U}_{\alpha}$ of $X_{\alpha}$ with $\operatorname{mesh}\left(\mathcal{U}_{\alpha}\right) \leq \tau$ and with Lebesgue number $L\left(\mathcal{U}_{\alpha}\right) \geq \delta \tau$.

For a metric space $X$ and $a>0$, we denote by $a X$ the metric space obtained from $X$ by multiplying all distances by $a$.

In Subsection 2.3, we recall the definitions of the asymptotic dimension asdim and the linearly controlled asymptotic dimension $\ell$-asdim, used in the following theorem; these are some quasi-isometry invariants of metric spaces.

Theorem 1.1. Let $X_{1}, X_{2}$ be visual Gromov hyperbolic spaces. Assume that for the metric spaces of the family $\left\{a \partial_{\infty} X_{1} \times b \partial_{\infty} X_{2}: a \geq 1, b \geq 1\right\}$ we have $\ell$-dim $\leq k$ uniformly, where the boundaries at infinity $\partial_{\infty} X_{1}, \partial_{\infty} X_{2}$ are taken with some visual metrics. Then

$$
\operatorname{asdim}\left(X_{1} \times X_{2}\right) \leq k+2
$$

The property of a Gromov hyperbolic space $X$ to be visual is a rough version of the property that every point $x \in X$ lies on a geodesic ray emanating from a fixed point $x_{0} \in X$; for the precise definition see $\S 2$.

Remark 1.2. A similar result is true in the case of an arbitrary finite number (more than two) of factors, and moreover, the estimate remains valid if we replace asdim by $\ell$-asdim. In other words, the following is true.

2000 Mathematics Subject Classification. Primary 54F45.

Key words and phrases. Asymptotic dimension, hyperbolic groups, linearly controlled dimension, quasi-isometry invariants.

Supported by RFBR (grant no. 05-01-00939). 
Let $X_{1}, \ldots, X_{n}$ be visual Gromov hyperbolic spaces. Assume that for the metric spaces of the family $\left\{a_{1} \partial_{\infty} X_{1} \times \cdots \times a_{n} \partial_{\infty} X_{n}: a_{i} \geq 1, i \in\{1, \ldots, n\}\right\}$ we have $\ell$-dim $\leq k$ uniformly. Then

$$
\operatorname{asdim}\left(X_{1} \times \cdots \times X_{n}\right) \leq k+n
$$

and

$$
\ell-\operatorname{asdim}\left(X_{1} \times \cdots \times X_{n}\right) \leq k+n
$$

For simplicity of notation, we shall consider the case of two spaces and the asymptotic dimension asdim.

Theorem 1.3. Let $X_{1}, \ldots, X_{n}$ be geodesic Gromov hyperbolic spaces. Then

$$
\operatorname{asdim}\left(X_{1} \times \cdots \times X_{n}\right) \geq \ell-\operatorname{dim}\left(\partial_{\infty} X_{1} \times \cdots \times \partial_{\infty} X_{n} \times[0,1]^{n}\right) .
$$

Corollary 1.4. Let $\Gamma_{1}, \Gamma_{2}$ be Gromov hyperbolic groups. Then

$$
\operatorname{asdim}\left(\Gamma_{1} \times \Gamma_{2}\right)=\operatorname{dim}\left(\partial_{\infty} \Gamma_{1} \times \partial_{\infty} \Gamma_{2}\right)+2 .
$$

As an application of this corollary, we

1) give examples of strict inequality in the product theorem for the asymptotic dimension in the class of hyperbolic groups (cf. [Dr3, Problem 23]; the corresponding example in the class of general metric spaces was given in $[\mathrm{BL}])$;

2) give examples of strict inequality in the product theorem for the hyperbolic dimension;

3) prove that $\operatorname{asdim}(\Gamma \times \mathbb{R})=\operatorname{asdim}(\Gamma)+1$ for any hyperbolic group $\Gamma$.

The above identity fails for general metric spaces. An example of a metric space $X$ of bounded geometry with finite asymptotic dimension for which $\operatorname{asdim}(X \times \mathbb{R})=\operatorname{asdim} X$ was constructed in [Dr4], and the question is whether the identity in 3) is true for groups ([Dr3, Problem 21]).

Theorem 1.5. Let $X$ be a visual Gromov hyperbolic space. Then

$$
\ell-\operatorname{asdim} X \leq \ell-\operatorname{dim}\left(\partial_{\infty} X \times[0,1]\right) .
$$

This refines the estimate $\operatorname{asdim} X \leq \ell-\operatorname{dim} \partial_{\infty} X+1$ proved in [Bu1, Bu2]. Unfortunately, we do not know whether the space $[0,1]$ is dimensionally full for the linearly controlled dimension, i.e., whether $\ell$-dim $(Y \times[0,1])=\ell$-dim $Y+1$ for any (compact or proper) metric space $Y$.

Corollary 1.6. Let $X$ be a visual, geodesic Gromov hyperbolic space. Then

$$
\ell-\operatorname{asdim} X=\operatorname{asdim} X=\ell-\operatorname{dim}\left(\partial_{\infty} X \times[0,1]\right) .
$$

The first identity fails in general, and the question is whether it holds for all finitely presented groups ([Dr3, Problem 41]).

\section{§2. Preliminaries}

Here, we recall some notions and facts necessary for the paper.

2.1. Coverings. Let $Z$ be a metric space. For $U, U^{\prime} \subset Z$, we denote by $\operatorname{dist}\left(U, U^{\prime}\right)$ the distance between $U$ and $U^{\prime}, \operatorname{dist}\left(U, U^{\prime}\right)=\inf \left\{\left|u u^{\prime}\right|: u \in U, u^{\prime} \in U^{\prime}\right\}$, where $\left|u u^{\prime}\right|$ is the distance between $u, u^{\prime}$. For $r>0$ we denote by $B_{r}(U)$ the open $r$-neighborhood of $U, B_{r}(U)=\{z \in Z: \operatorname{dist}(z, U)<r\}$, and by $\bar{B}_{r}(U)$ the closed $r$-neighborhood of $U, \bar{B}_{r}(U)=\{z \in Z: \operatorname{dist}(z, U) \leq r\}$. We extend this notation to all real $r$ by putting $B_{r}(U)=U$ for $r=0$ and by defining $B_{r}(U)$ for $r<0$ as the complement to the closed $|r|$-neighborhood of $Z \backslash U, B_{r}(U)=Z \backslash \bar{B}_{|r|}(Z \backslash U)$. 
Given a family $\mathcal{U}$ of subsets in a metric space $Z$, we define $\operatorname{mesh}(\mathcal{U})=\sup \{\operatorname{diam} U$ : $U \in \mathcal{U}\}$. The multiplicity $m(\mathcal{U})$ of $\mathcal{U}$ is the maximal number of the members of $\mathcal{U}$ with nonempty intersection. We say that a family $\mathcal{U}$ is disjoint if $m(\mathcal{U})=1$.

A family $\mathcal{U}$ is called a covering of $Z$ if $\bigcup\{U: U \in \mathcal{U}\}=Z$. A covering $\mathcal{U}$ is said to be colored if it is the union of $m \geq 1$ disjoint families, $\mathcal{U}=\bigcup_{a \in A} \mathcal{U}^{a},|A|=m$. In this case we also say that $\mathcal{U}$ is $m$-colored. Clearly, the multiplicity of an $m$-colored covering is at most $m$.

Let $\mathcal{U}$ be a family of open subsets in a metric space $Z$ that cover $A \subset Z$. Given $z \in A$, we let

$$
L(\mathcal{U}, z)=\sup \{\operatorname{dist}(z, Z \backslash U): U \in \mathcal{U}\}
$$

be the Lebesgue number of $\mathcal{U}$ at $z$, and $L(\mathcal{U})=\inf _{z \in A} L(\mathcal{U}, z)$ the Lebesgue number of the covering $\mathcal{U}$ of $A$. For every $z \in A$, the open ball $B_{r}(z) \subset Z$ of radius $r=L(\mathcal{U})$ centered at $z$ is contained in some member of the covering $\mathcal{U}$.

We shall use the following obvious fact (see, e.g., [Bu1]).

Lemma 2.1. Let $\mathcal{U}$ be an open covering of $A \subset Z$ with $L(\mathcal{U})>0$. Then for every $s \in(0, L(\mathcal{U}))$ the family $\mathcal{U}_{-s}=B_{-s}(\mathcal{U})$ is still an open covering of $A$.

2.2. Hyperbolic spaces. Here we recall the necessary facts from the theory of hyperbolic spaces. For more details, we refer the reader, e.g., to [BoS]. We also assume that the reader is familiar with notions such as a geodesic metric space, a geodesic ray, etc.

Let $X$ be a metric space. We use the notation $\left|x x^{\prime}\right|$ for the distance between $x, x^{\prime} \in X$. For $o \in X$ and $x, x^{\prime} \in X$, put $\left(x \mid x^{\prime}\right)_{o}=\frac{1}{2}\left(|x o|+\left|x^{\prime} o\right|-\left|x x^{\prime}\right|\right)$. The number $\left(x \mid x^{\prime}\right)_{o}$ is nonnegative by the triangle inequality; it is called the Gromov product of $x, x^{\prime}$ with respect to $o$.

A metric space $X$ is said to be $\delta$-hyperbolic, $\delta \geq 0$, if the inequality

$$
(x \mid y)_{o} \geq \min \left\{(x \mid z)_{o},(z \mid y)_{o}\right\}-\delta
$$

is fulfilled for every base point $o \in X$ and all $x, y, z \in X$.

Let $X$ be a hyperbolic space, i.e., $X$ is $\delta$-hyperbolic for some $\delta \geq 0$. We denote by $\partial_{\infty} X$ the (Gromov) boundary of $X$ at infinity. The Gromov product based at $o \in X$ extends naturally to $\partial_{\infty} X$.

If a hyperbolic space $X$ is geodesic, then every geodesic ray in $X$ represents a point at infinity. Conversely, if a geodesic hyperbolic space $X$ is proper (i.e., the closed balls in $X$ are compact), then every point at infinity is represented by a geodesic ray.

A metric $d$ on the boundary at infinity $\partial_{\infty} X$ of $X$ is said to be visual if there are $o \in X, a>1$, and positive constants $c_{1}, c_{2}$, such that

$$
c_{1} a^{-\left(\xi \mid \xi^{\prime}\right)_{o}} \leq d\left(\xi, \xi^{\prime}\right) \leq c_{2} a^{-\left(\xi \mid \xi^{\prime}\right)_{o}}
$$

for all $\xi, \xi^{\prime} \in \partial_{\infty} X$. In this case, we say that $d$ is visual with respect to the base point $o$ and the parameter $a$. The boundary at infinity is bounded and complete with respect to any visual metric; moreover, if $X$ is proper, then $\partial_{\infty} X$ is compact. If $a>1$ is sufficiently close to 1 , then a visual metric with respect to $a$ does exist.

A hyperbolic space $X$ is visual if for some base point $x_{0} \in X$ there is a positive constant $D$ such that for every $x \in X$ there is $\xi \in \partial_{\infty} X$ with $\left|x x_{0}\right| \leq(x \mid \xi)_{x_{0}}+D$ (it is easily seen that this property is independent of the choice of $x_{0}$ ).

2.3. Definitions of the linearly controlled and the asymptotic dimension. We shall say that a covering $\mathcal{U}$ is an $(L, M, n)$-covering if it is $n$-colored with $\operatorname{mesh}(\mathcal{U}) \leq M$ and $L(\mathcal{U}) \geq L$.

The notion of the linearly controlled metric dimension of a metric space was introduced in [Bu1], where it was called the "capacity dimension". One of many equivalent definitions of this notion is the following. 
The linearly controlled dimension, or $\ell$-dimension, of a metric space $Z$, $\ell$ - $\operatorname{dim}(Z)$, is the minimal integer $m \geq 0$ with the following property: there is a constant $\delta \in(0,1)$ such that for every sufficiently small $\tau>0$ there exists an open $(\delta \tau, \tau, m+1)$-covering of $Z$. Here (and in all similar definitions of a dimension below) we assume that $\ell$-dim $Z=\infty$ if there is no $m$ with the property indicated.

The asymptotic dimension is a quasi-isometry invariant of a metric space; it was introduced in $[\mathrm{Gr}]$. There are also several equivalent definitions; see $[\mathrm{Gr}],[\mathrm{BD}]$. We use the following one. The asymptotic dimension of a metric space $X$ is the minimal integer $\operatorname{asdim} X=m$ such that for every positive $d$ there is an open covering $\mathcal{U}$ of $X$ with $m(\mathcal{U}) \leq m+1, \operatorname{mesh}(\mathcal{U})<\infty$, and $L(\mathcal{U}) \geq d$.

The linearly controlled asymptotic dimension is also a quasi-isometry invariant of a metric space. We use the following definition. The linearly controlled asymptotic dimension of a metric space $X$ is the minimal integer $\ell$-asdim $X=n$ with the following property: there is a constant $C>1$ such that for every sufficiently large $d$ there is an open $(d, C d, n+1)$-covering $\mathcal{U}$ of $X$.

2.4. Coverings of the product. Throughout the paper, we assume that the product of metric spaces $X \times Y$ is endowed with the $l_{\infty}$-metric; i.e., $\left|(x, y)\left(x^{\prime}, y^{\prime}\right)\right|=\max \left\{\left|x x^{\prime}\right|,\left|y y^{\prime}\right|\right\}$ for each $(x, y),\left(x^{\prime}, y^{\prime}\right) \in X \times Y$.

Let $\mathcal{U}$ be an open covering of the product $X \times Y$ of metric spaces. We say that a pair $(p, q), p, q \geq 0$, dominates $\operatorname{mesh}^{\times}(\mathcal{U})$ and write $\operatorname{mesh}^{\times}(\mathcal{U}) \leq(p, q)$ or $(p, q)=\operatorname{mesh}^{\bigotimes}(\mathcal{U})$ if for each member of $\mathcal{U}$ there exists a point $(x, y) \in X \times Y$ such that this member is contained in the product $\bar{B}_{p}(x) \times \bar{B}_{q}(y)$.

We also say that a function $f(z) \in[0, \infty) \times[0, \infty), z \in X \times Y$, dominates the pointwise $\operatorname{mesh}^{\times}(\mathcal{U}, z)$ and write $\operatorname{mesh}^{\times}(\mathcal{U}, z) \leq f(z)$ or $f(z)=\operatorname{mesh}^{\bigotimes}(\mathcal{U}, z)$ if for each member of $\mathcal{U}$ that contains $z$ there exists a point $(x, y) \in X \times Y$ such that this member is contained in $\bar{B}_{p}(x) \times \bar{B}_{q}(y)$, where $(p, q)=f(z)$. In this sense, the mesh ${ }^{\times}$(the pointwise mesh ${ }^{\times}$) is determined basically by the meshes (the meshes at the projected points) of the coverings projected to the factors.

Similarly, we say that the Lebesgue number $L^{\times}$at a point $z=(x, y) \in X \times Y$ of the covering $\mathcal{U}$ dominates $f(z)=(p, q) \in[0, \infty) \times[0, \infty), L^{\times}(\mathcal{U}, z) \geq f(z)$, if the product of the balls $\bar{B}_{p}(x) \times \bar{B}_{q}(y)$ is contained in some member of the covering. In this case, we also write $f(z)=L^{\otimes}(\mathcal{U}, z)$.

We write $L^{\times}(\mathcal{U}) \geq(p, q)$ or $(p, q)=L^{\bigotimes}(\mathcal{U})$ if $L^{\times}(\mathcal{U}, z) \geq(p, q)$ for every $z \in X \times Y$. In general, there is no way to recover $L^{\times}$by the Lebesgue numbers of the projected coverings.

For the product $X \times Y$, it is straightforward to check that the following two properties are equivalent.

(1) The spaces of the family $\{a X \times b Y: a \geq 1, b \geq 1\}$ have $\ell$-dim $\leq k$ uniformly.

(2) There is a constant $\delta \in(0,1)$ such that for every sufficiently small $\tau>0$ and every $\alpha, \beta \in(0,1]$ there exists a $(k+1)$-colored open covering $\mathcal{U}$ of $X \times Y$ with $\operatorname{mesh}^{\times}(\mathcal{U}) \leq$ $(\alpha \tau, \beta \tau)$ and $L^{\times}(\mathcal{U}) \geq(\alpha \delta \tau, \beta \delta \tau)$.

Let $\mathcal{U}, \mathcal{V}$ be open coverings of $X \times Y$. We write $\operatorname{mesh}^{\times}(\mathcal{U}) \leq(p, q) L^{\times}(\mathcal{V})$ for some $p, q \geq 0$ if $\operatorname{mesh}^{\times}(\mathcal{U}) \leq\left(p r_{1}, q r_{2}\right)$ for some $\left(r_{1}, r_{2}\right)=L^{\bigotimes}(\mathcal{V})$. If $p=q$, we write $\operatorname{mesh}^{\times}(\mathcal{U}) \leq p L^{\times}(\mathcal{V})$. Observe that if $\operatorname{mesh}^{\times}(\mathcal{U}) \leq L^{\times}(\mathcal{V})$, then the covering $\mathcal{U}$ is inscribed in the covering $\mathcal{V}$; that is, every member of $\mathcal{U}$ is contained in some member of $\mathcal{V}$.

We write $\operatorname{mesh}^{\times}(\mathcal{U}, z) \leq \operatorname{mesh}^{\times}(\mathcal{V}, z)$ if every pair mesh ${ }^{\bigotimes}(\mathcal{V}, z)$ is a pair $\operatorname{mesh}^{\bigotimes}(\mathcal{U}, z)$. We write $L^{\times}(\mathcal{U}, z) \geq s L^{\times}(\mathcal{V}, z)$ for $s \geq 0$ if for any $p, q \geq 0$ with $L^{\times}(\mathcal{V}, z) \geq(p, q)$ we have $L^{\times}(\mathcal{U}, z) \geq(s p, s q)$. 
For $A \subset Z=X \times Y$ and $r_{1}, r_{2}>0$, we denote by $B_{\left(r_{1}, r_{2}\right)}(A)$ the union of all products $B_{r_{1}}(x) \times B_{r_{2}}(y)$ with $(x, y) \in A, B_{\left(r_{1}, r_{2}\right)}(A)=\bigcup\left\{B_{r_{1}}(x) \times B_{r_{2}}(y):(x, y) \in A\right\}$, and put $B_{\left(-r_{1},-r_{2}\right)}(A)=Z \backslash \bar{B}_{\left(r_{1}, r_{2}\right)}(Z \backslash A)$.

We also write $(p, q) \geq\left(p^{\prime}, q^{\prime}\right)$ for reals $p, q, p^{\prime}, q^{\prime}$ if and only if $p \geq p^{\prime}$ and $q \geq q^{\prime}$.

\section{§3. Auxiliary facts}

3.1. Separate and qualified families of coverings. In this section we give a generalization of the following lemma, the proof of which can be found, e.g., in [BL].

Lemma 3.1. Suppose that $Z$ is a metric space and $A, B \subset Z$. Let $\mathcal{U}$ be an open covering of $A, \mathcal{V}$ an open covering of $B$, both of multiplicity at most $m$. If $\operatorname{mesh}(\mathcal{V}) \leq$ $L(\mathcal{U}) / 2$, then there exists an open covering $\mathcal{W}$ of $A \cup B$ with multiplicity at most $m$ and $\operatorname{mesh}(\mathcal{W}) \leq \max \{\operatorname{mesh}(\mathcal{V}), \operatorname{mesh}(\mathcal{U})\}, L(\mathcal{W}) \geq \min \{L(\mathcal{U}) / 2, L(\mathcal{V})\}$

Let $\mathcal{U}_{1}, \mathcal{U}_{2}$ be families of subsets in a metric space $Z$. We say that $\mathcal{U}_{1}$ and $\mathcal{U}_{2}$ are separate if every member of $\mathcal{U}_{1}$ intersects no member of $\mathcal{U}_{2}$.

Let $Z=X \times Y$ be the product of metric spaces, and let $Z_{\alpha} \subset Z, \alpha \in \mathcal{A}$. Let $\mathcal{U}_{\alpha}$ be a covering of $Z_{\alpha}$. Let $S=\{0, \ldots, N\}$ be the set of "scales" and $i: \mathcal{A} \rightarrow S$ a scale function.

We say that the family $\mathcal{U}_{\alpha}, \alpha \in \mathcal{A}$, is separate with the scale function $i$ if for every $U \in \mathcal{U}_{\alpha}, V \in \mathcal{U}_{\alpha^{\prime}}$ with $\alpha \neq \alpha^{\prime}$ and $i(\alpha)=i\left(\alpha^{\prime}\right)$, we have $U \cap V=\varnothing$.

We say that the family $\mathcal{U}_{\alpha}$ is qualified by the scale function $i$ if the condition $U \cap V \neq \varnothing$ for some $U \in \mathcal{U}_{\alpha}, V \in \mathcal{U}_{\alpha^{\prime}}$ with $i(\alpha)<i\left(\alpha^{\prime}\right)$ implies $L^{\bigotimes}\left(\mathcal{U}_{\alpha^{\prime}}\right) \geq 4 \operatorname{mesh}^{\bigotimes}\left(\mathcal{U}_{\alpha}\right)$ for appropriate dominating numbers fixed for each covering $\mathcal{U}_{\alpha}$.

Proposition 3.2. Suppose that $Z=X \times Y$ is the product of metric spaces and $Z_{\alpha} \subset Z$, $\alpha \in \mathcal{A}$. Let $S=\{0, \ldots, N\}$, and let $i: \mathcal{A} \rightarrow S$ be a scale function. Let $\mathcal{U}_{\alpha}$ be an open $m$-colored covering of $Z_{\alpha}, \alpha \in \mathcal{A}$, such that the family of coverings $\mathcal{U}_{\alpha}, \alpha \in \mathcal{A}$, is separate with and qualified by the scale function $i$. Then there exists an open $m$-colored covering $\mathcal{W}$ of $\bigcup_{\alpha} Z_{\alpha}$ with $\operatorname{mesh}^{\times}(\mathcal{W}, z) \leq \operatorname{mesh}^{\times}\left(\bigcup_{\alpha} \mathcal{U}_{\alpha}, z\right)$ and $L^{\times}(\mathcal{W}, z) \geq L^{\times}\left(\bigcup_{\alpha} \mathcal{U}_{\alpha}, z\right) / 2$ for every $z \in \bigcup_{\alpha} Z_{\alpha}$.

Proof. Let $A$ be the set of colors, $|A|=m$, which may be assumed to be common for all coverings $\mathcal{U}_{\alpha}$, so that $\mathcal{U}_{\alpha}$ is the union of disjoint families, $\mathcal{U}_{\alpha}=\bigcup_{a \in A} \mathcal{U}_{\alpha}^{a}$. For $s \in S$, we denote $\mathcal{A}_{s}:=\{\alpha \in \mathcal{A}: i(\alpha)=s\}$.

For every $\alpha \in \mathcal{A}$ and $a \in A$, we consider the family $\mathcal{V}_{\alpha}^{a}=B_{-L^{\otimes}\left(\mathcal{U}_{\alpha}\right) / 2}\left(\mathcal{U}_{\alpha}^{a}\right)$, where the pair $L^{\bigotimes}\left(\mathcal{U}_{\alpha}\right)$ is dominated by $L^{\times}\left(\mathcal{U}_{\alpha}\right)$ as in the definition of coverings qualified by a scale function.

Assume that $U \in \mathcal{U}_{\alpha}$ and $V=B_{-L^{凶}\left(\mathcal{U}_{\alpha}\right) / 2}\left(V^{\prime}\right)$ for $V^{\prime} \in \mathcal{U}_{\alpha^{\prime}}^{a}$. Then

(*) for $i(\alpha)<i\left(\alpha^{\prime}\right)$ and $U \cap V \neq \varnothing$, we have $U \cup V \subset V^{\prime}$.

This easily follows from the fact that $L^{\bigotimes}\left(\mathcal{U}_{\alpha^{\prime}}\right) \geq 4$ mesh $^{\bigotimes}\left(\mathcal{U}_{\alpha}\right)$ in this case. In particular,

(**) for every $U \in \mathcal{U}_{\alpha}$, every $\alpha^{\prime} \in \mathcal{A}$ with $i(\alpha)<i\left(\alpha^{\prime}\right)$, and every $a \in A$ there exists at most one $V \in \mathcal{V}_{\alpha^{\prime}}^{a}$ with $U \cap V \neq \varnothing$,

because the family $\mathcal{U}_{\alpha^{\prime}}^{a}$ is disjoint.

We construct an $m$-colored covering $\mathcal{W}$ by induction on $j \in S$, independently for each color $a \in A$.

Fix a color $a \in A$ and set $\mathcal{W}_{0}^{a}:=\bigcup_{\alpha \in \mathcal{A}_{0}} U_{\alpha}^{a}$. Then the family $\mathcal{W}_{0}^{a}$ is disjoint; moreover, it possesses properties (2), (3) below. Assume that for some $j \in S$ we have already constructed a family $\mathcal{W}_{j}^{a}$ with the following properties:

(1) $\mathcal{W}_{j}^{a}$ is disjoint;

(2) for every $U \in \mathcal{V}_{\alpha}^{a}$ with $i(\alpha) \leq j$ there exists $W \in \mathcal{W}_{j}^{a}$ such that $U \subset W$;

(3) for every $W \in \mathcal{W}_{j}^{a}$ there exists $U \in \mathcal{U}_{\alpha}^{a}$ with $i(\alpha) \leq j$ such that $W \subset U$. 
In view of $(* *)$, property (3) implies that for every $W \in \mathcal{W}_{j}^{a}$ there exists at most one member $V \in \bigcup_{\mathcal{A}_{j+1}} \mathcal{V}_{\alpha}^{a}$ such that $V \cap W \neq \varnothing$. We set $I(W)=V$ if such a $V$ exists and $I(W)=\varnothing$ otherwise; this yields a function $I: \mathcal{W}_{j}^{a} \rightarrow \bigcup_{\mathcal{A}_{j+1}} \mathcal{V}_{\alpha}^{a} \cup\{\varnothing\}$.

We put

$$
\mathcal{W}_{j+1}^{a}=\left\{W \in \mathcal{W}_{j}^{a}: I(W)=\varnothing\right\} \cup\left\{\left(V \cup I^{-1}(V): V \in \bigcup_{\mathcal{A}_{j+1}} \mathcal{V}_{\alpha}^{a}\right\}\right.
$$

The family $\bigcup_{\mathcal{A}_{j+1}} \mathcal{V}_{\alpha}^{a}$ is disjoint. Thus, by (*) and (1), the family $\mathcal{W}_{j+1}^{a}$ is also disjoint. Furthermore, $\mathcal{W}_{j+1}^{a}$ possesses property (2) (with $j$ replaced with $j+1$ ) by construction. Property (3) is also fulfilled for $\mathcal{W}_{j+1}^{a}$ by $(*)$ and the inductive assumption.

Proceeding by induction, we obtain a family $\mathcal{W}_{N}^{a}$ for each $a \in A$. We set $\mathcal{W}^{a}=$ $\mathcal{W}_{N}^{a}$ and $\mathcal{W}=\bigcup_{a} \mathcal{W}^{a}$. Property $(2)$ for $\mathcal{W}_{N}^{a}$ implies that $\mathcal{W}$ is a covering of $\bigcup_{\alpha} Z_{\alpha}$. By property (1) for $\mathcal{W}_{N}^{a}, \mathcal{W}$ is $m$-colored. Property (3) implies that $\operatorname{mesh}^{\times}(\mathcal{W}, z) \leq$ mesh $^{\times}\left(\bigcup_{\alpha} \mathcal{U}_{\alpha}, z\right)$ and property $(2)$ implies that $L^{\times}(\mathcal{W}, z) \geq L^{\times}\left(\bigcup_{\alpha} \mathcal{U}_{\alpha}, z\right) / 2$ for every $z \in \bigcup_{\alpha} Z_{\alpha}$. That is, $\mathcal{W}$ possesses all the required properties.

3.2. Barycentric triangulation of products. We recall some standard constructions related to simplicial polyhedra. Given an index set $J$, we let $R^{J}$ be the Euclidean space of functions $J \rightarrow \mathbb{R}$ with finite support. For $x, x^{\prime} \in \mathbb{R}^{J}$, the distance $\left|x x^{\prime}\right|$ is well defined by

$$
\left|x x^{\prime}\right|^{2}=\sum_{j \in J}\left(x_{j}-x_{j}^{\prime}\right)^{2} .
$$

Let $\Delta^{J} \subset R^{J}$ be the standard simplex; i.e., $x \in \Delta^{J}$ if and only if $x_{j} \geq 0$ for all $j \in J$ and $\sum_{\in J} x_{j}=1$. The metric of $R^{J}$ induces a metric on $\Delta^{J}$ and on every subcomplex $K \subset \Delta^{J}$. If $J$ is finite, then $|J|-1=\operatorname{dim} \Delta^{J}$ is the (combinatorial) dimension of $\Delta^{J}$.

For every simplicial polyhedron $K$, there is a canonical embedding $u: K \rightarrow \Delta^{J}$, where $J$ is the vertex set of $K$, which is affine on every simplex. Its image $K^{\prime}=u(K)$ is called the uniformization of $K$. The (combinatorial) dimension of $K$ is the maximal dimension of its simplexes.

For a vertex $v \in K$, its star $\overline{\operatorname{st}}_{v} \subset K$ consists of all simplexes of $K$ containing $v$. The open star st $v$ of $v$ is the star without the faces that miss $v$. If a simplicial polyhedron $K$ is uniform, then the open star st ${ }_{v}$ of any vertex $v \in K$ is an open neighborhood of $v$.

There are several ways to triangulate the product of simplicial complexes. For that, one needs to choose some ordering of simplexes. Since the barycentric triangulation is ordered canonically, we prefer to use the following construction, which can be found, e.g., in the forthcoming book [BS3]. Given an index set $J$, we denote by $\overline{\mathrm{ba}} \Delta^{J}$ the barycentric subdivision of $\Delta^{J}$, which is a simplicial complex isometric to $\Delta^{J}$. The vertices of this complex are barycenters of all simplexes (including the 0-dimensional ones). The simplexes of this complex are the convex hulls of all sets $S$ of vertices with the following property: if $s, s^{\prime} \in S$, then they are the barycenters of two simplexes, one of which is contained in the other. If $J$ is finite, then the covering of $\Delta^{J}$ by the open stars of the vertices of its barycentric subdivision is $|J|$-colored: as the color of a star st $_{v}$, we can take the dimension of the face for which $v$ is the barycenter.

Now, we describe the barycentric triangulation of the product of two simplexes $\Delta^{J_{1}}$, $\Delta^{J_{2}}$. We regard this product $\Delta^{J_{1}} \times \Delta^{J_{2}}$ as a complex with faces that are products of standard simplexes. Let $b_{1}, b_{2}$ be barycenters of simplexes $S_{1} \subset \Delta^{J_{1}}, S_{2} \subset \Delta^{J_{2}}$. We call the point $\left(b_{1}, b_{2}\right) \in \Delta^{J_{1}} \times \Delta^{J_{2}}$ the barycenter of the face $S_{1} \times S_{2}$. We define the barycentric subdivision of $\Delta^{J_{1}} \times \Delta^{J_{2}}$ as a simplicial complex $\Delta^{J_{1}} \overline{\times}_{s} \Delta^{J_{2}}$, isometric to $\Delta^{J_{1}} \times \Delta^{J_{2}}$, in the following way. The vertices of this complex are the barycenters of all faces (including the 0 -dimensional ones). The simplexes of this complex are the convex 
hulls of all sets $S$ of vertices with the following property: if $s, s^{\prime} \in S$, then they are the barycenters of two faces, one of which is contained in the other.

We denote the uniformization of $\Delta^{J_{1}} \overline{\times}_{s} \Delta^{J_{2}}$ by $\Delta^{J_{1}} \times{ }_{s} \Delta^{J_{2}}$ and call it the barycentric triangulation of the product $\Delta^{J_{1}} \times \Delta^{J_{2}}$.

The canonical bijection $\phi: \Delta^{J_{1}} \times \Delta^{J_{2}} \rightarrow \Delta^{J_{1}} \times{ }_{s} \Delta^{J_{2}}$ is called the barycentric triangulation map.

Now, let $K_{1}, K_{2}$ be uniform simplicial polyhedra, which we can identify with subpolyhedra of the standard simplexes $\Delta^{J_{1}}, \Delta^{J_{2}}$; we define the barycentric triangulation of $K_{1} \times K_{2}$ as $\phi\left(K_{1} \times K_{2}\right)$.

Note that the covering of $K_{1} \times{ }_{s} K_{2}$ by open stars of its vertices is $\left(n_{1}+n_{2}+1\right)$-colored, where $n_{1}=\operatorname{dim} K_{1}, n_{2}=\operatorname{dim} K_{2}$ : as the color of a star st $v$, we can take the dimension of the minimal simplex of $\Delta^{J_{1} \cup J_{2}}$ containing $v$.

We give the following two technical lemmas without proof (for the proof, see [BS3]).

Lemma 3.3. Let $K_{1}, K_{2}$ be uniform simplicial polyhedra both of finite dimension. The barycentric triangulation map

$$
\phi: K_{1} \times K_{2} \rightarrow K_{1} \times{ }_{s} K_{2}
$$

is bi-Lipschitz, with bi-Lipschitz constant depending only on the dimensions of $K_{1}$ and $K_{2}$.

Lemma 3.4. Let $K_{1}, K_{2}$ be uniform simplicial polyhedra, and let $K=K_{1} \times_{s} K_{2}$. For every vertex $v \in K$, there are vertices $v_{1} \in K_{1}$ and $v_{2} \in K_{2}$ such that $\phi^{-1}\left(s t_{v}\right) \subset$ $s t_{v_{1}} \times s t_{v_{2}}$.

Recall that the nerve $\mathcal{N}(\mathcal{U})$ of a covering $\mathcal{U}=\left\{U_{j}\right\}_{j \in J}$ is the simplicial complex whose vertices are the members of the covering and a collection of vertices spans a simplex if and only if the corresponding members of $\mathcal{U}$ have nonempty intersection. We assume that the nerves we consider are uniform polyhedra, unless the opposite is stated explicitly; moreover, we can regard $\mathcal{N}(\mathcal{U})$ as a subcomplex of $\Delta^{J}$.

Let $\mathcal{U}$ be an open locally finite covering of a metric space $Z$ such that no member of $\mathcal{U}$ coincides with $Z$. The barycentric map $p: Z \rightarrow \mathcal{N}(\mathcal{U})$ is defined as follows. For every $j \in J$ we put $q_{j}(z)=\operatorname{dist}\left(z, Z \backslash U_{j}\right)$. Since the covering is open, $\sum_{j \in J} q_{j}(z)>0$. Since no member of $\mathcal{U}$ coincides with $Z$ and the covering is locally finite, $\sum_{j \in J} q_{j}(z)<\infty$ for every point $z \in Z$. Then we define the coordinate functions of the map $p: Z \rightarrow \mathbb{R}^{J}$ by $p_{j}(z)=q_{j}(z) / \sum_{j \in J} q_{j}(z)$. Clearly, $p(Z) \subset \mathcal{N}(\mathcal{U})$. For every vertex $v \in \mathcal{N}(\mathcal{U})$, the preimage $p^{-1}\left(\mathrm{st}_{v}\right)$ coincides with the corresponding element of the covering.

Suppose, moreover, that $m(\mathcal{U}) \leq m+1$ and $L(\mathcal{U}) \geq d>0$. Then, it is not difficult to prove (see, e.g., [BS1]) that the map $p$ is Lipschitz with Lipschitz constant

$$
\operatorname{Lip}(p) \leq(m+2)^{2} / d .
$$

Lemma 3.5. Let $X_{1}, X_{2}$ be metric spaces. Let $\mathcal{U}_{1}$ be an open covering of $X_{1}$ and $\mathcal{U}_{2}$ an open covering of $X_{2}$, of multiplicities at most $n_{1}+1$ and $n_{2}+1$, respectively. Then there exists an $\left(n_{1}+n_{2}+1\right)$-colored open covering $\mathcal{W}$ of $X_{1} \times X_{2}$ with

$$
L^{\times}(\mathcal{W}) \geq q\left(L\left(\mathcal{U}_{1}\right), L\left(\mathcal{U}_{2}\right)\right) \quad \text { and } \quad \operatorname{mesh}^{\times}(\mathcal{W}) \leq\left(\operatorname{mesh}\left(\mathcal{U}_{1}\right), \operatorname{mesh}\left(\mathcal{U}_{2}\right)\right),
$$

where the constant $q$ depends only on $n_{1}, n_{2}$.

Proof. In accordance with the definitions of $L^{\times}$and mesh $^{\times}$, rescaling the metric of $X_{1} \times X_{2}$ in one of the factors does not change the problem; i.e., if we construct a required covering for, say, $a X_{1} \times X_{2}$, then rescaling the metric back gives a required covering for $X_{1} \times X_{2}$. 
So, by rescaling of the metric, we may assume that $L\left(\mathcal{U}_{2}\right)=\frac{\left(n_{2}+2\right)^{2}}{\left(n_{1}+2\right)^{2}} L\left(\mathcal{U}_{1}\right)$. Denote

$$
\lambda:=\frac{\left(n_{1}+2\right)^{2}}{L\left(\mathcal{U}_{1}\right)}=\frac{\left(n_{2}+2\right)^{2}}{L\left(U_{2}\right)} .
$$

Let $K_{i}$ be the nerve of $U_{i}$ and $p_{i}: X_{i} \rightarrow K_{i}$ the corresponding barycentric map, $i=1,2$. We have

$$
\operatorname{Lip}\left(p_{i}\right) \leq \lambda
$$

Then for the map $p: X_{1} \times X_{2} \rightarrow K_{1} \times K_{2}$ defined by $p\left(x_{1}, x_{2}\right)=\left(p_{1}\left(x_{1}\right), p_{2}\left(x_{2}\right)\right)$, we have $\operatorname{Lip}(p) \leq \lambda$.

By Lemma 3.3, the barycentric triangulation map

$$
\phi: K_{1} \times K_{2} \rightarrow K_{1} \times{ }_{s} K_{2}
$$

is Lipschitz with Lipschitz constant $c$ depending only on $n_{1}, n_{2}$. Consider the covering $\mathcal{V}$ of $K_{1} \times{ }_{s} K_{2}$ by open stars of its vertices, which is $\left(n_{1}+n_{2}+1\right)$-colored. Since the polyhedron $K_{1} \times{ }_{s} K_{2}$ is uniform, the Lebesgue number of this covering is bounded from below by a positive constant $l$ depending only on $\operatorname{dim}\left(K_{1} \times{ }_{s} K_{2}\right)$.

Now, let $\mathcal{W}$ be the open covering of $X_{1} \times X_{2}$ by the preimages of the elements of $\mathcal{V}$ under the map $\phi \circ p$. Then $\mathcal{W}$ is $\left(n_{1}+n_{2}+1\right)$-colored and

$$
L(\mathcal{W}) \geq \lambda^{-1} c^{-1} l=\frac{l}{c\left(n_{1}+2\right)^{2}} L\left(\mathcal{U}_{1}\right)=\frac{l}{c\left(n_{2}+2\right)^{2}} L\left(\mathcal{U}_{2}\right) ;
$$

in particular, $L^{\times}(\mathcal{W}) \geq q\left(L\left(\mathcal{U}_{1}\right), L\left(\mathcal{U}_{2}\right)\right)$ with $q=\frac{l}{c \max \left\{\left(n_{1}+2\right)^{2},\left(n_{2}+2\right)^{2}\right\}}$.

By Lemma 3.4, for every $W \in \mathcal{W}$ there are $U_{1} \in \mathcal{U}_{1}$ and $U_{2} \in \mathcal{U}_{2}$ with $W \subset U_{1} \times U_{2}$, so that $\operatorname{mesh}^{\times}(\mathcal{W}) \leq\left(\operatorname{mesh} \mathcal{U}_{1}, \operatorname{mesh} \mathcal{U}_{2}\right)$. The lemma follows.

We denote the covering described in this lemma by $\mathcal{W}=\mathcal{U} * \mathcal{V}$. In the following corollary, we use the natural extension of our notation mesh ${ }^{\times}$and $L^{\times}$to the case of more than two factors.

Corollary 3.6. Let $\mathcal{U}_{i}$ be an open covering with multiplicity at most $n_{i}+1$ of a metric space $X_{i}, i \in\{1, \ldots, k\}$. We let $\mathcal{W}_{i}=\mathcal{W}_{i-1} * \mathcal{U}_{i}$ be the covering of $X_{1} \times \cdots \times X_{i}$ for $i=1, \ldots, k$, where $\mathcal{W}_{1}=\mathcal{U}_{1}$. Then $\mathcal{W}=\mathcal{W}_{k}$ is an open $m$-colored covering of $X_{1} \times \cdots \times X_{k}$ with $m=n_{1}+\cdots+n_{k}+1$,

$$
L^{\times}(\mathcal{W}) \geq q\left(L\left(\mathcal{U}_{1}\right), \ldots, L\left(\mathcal{U}_{k}\right)\right)
$$

and

$$
\operatorname{mesh}^{\times}(\mathcal{W}) \leq\left(\operatorname{mesh}\left(\mathcal{U}_{1}\right), \ldots, \operatorname{mesh}\left(\mathcal{U}_{k}\right)\right)
$$

where the constant $q$ depends only on $n_{1}, \ldots, n_{k}$.

Proof. Arguing as at the beginning of the above proof and rescaling the metrics of the factors, we may assume that $L\left(\mathcal{U}_{1}\right)=\cdots=L\left(\mathcal{U}_{k}\right)=r$.

Note that, for a covering $\mathcal{U}$ of a product, the property $L^{\times}(\mathcal{U}) \geq(r, \ldots, r)$ and the property $L(\mathcal{U}) \geq r$ are equivalent. Using this and proceeding by induction, we obtain $L^{\times}(\mathcal{W}) \geq q(r, \ldots, r)$ for some constant $q$ depending only on $n_{1}, \ldots, n_{k}$.

By Lemma 3.4, for every $W \in \mathcal{W}_{i}$ there are $W^{\prime} \in \mathcal{W}_{i-1}$ and $U \in \mathcal{U}_{i}$ such that $W \subset W^{\prime} \times U$, for every $i=2, \ldots, k$. Consequently,

$$
\operatorname{mesh}^{\times}(\mathcal{W}) \leq\left(\operatorname{mesh} \mathcal{U}_{1}, \ldots, \operatorname{mesh} \mathcal{U}_{k}\right) .
$$

We shall need the following fact.

Lemma 3.7. Let $X, X_{1}$ and $X_{2}$ be metric spaces. 
(1) Assume that $\ell-\operatorname{dim}(X \times[0,1]) \leq k$. Then there exist $\tau_{0}>0$ and $\sigma>1$ such that for every $0<\tau<\tau_{0}$ and $\tau^{\prime}>0$ there exists a $(k+1)$-colored open covering $\mathcal{U}$ of $X \times \mathbb{R}$ with $\operatorname{mesh}^{\times}(\mathcal{U}) \leq\left(\sigma \tau, \sigma \tau^{\prime}\right)$ and $L^{\times}(\mathcal{U}) \geq\left(\tau, \tau^{\prime}\right)$.

(2) Assume that for the spaces of the family $a X_{1} \times b X_{2}$ we have $\ell$-dim $\leq k$ uniformly in $a, b \geq 1$. Then there exist $\tau_{0}>0$ and $\sigma>1$ such that for every $0<$ $\tau_{1}, \tau_{2}<\tau_{0}$ and $\tau_{1}^{\prime}, \tau_{2}^{\prime}>0$ there exists a $(k+3)$-colored open covering $\mathcal{W}$ of $Z=X_{1} \times X_{2} \times \mathbb{R} \times \mathbb{R}$ with $\operatorname{mesh}^{\times}(\mathcal{W}) \leq \sigma \tau, L^{\times}(\mathcal{W}) \geq \tau$, where $\tau=\left(\tau_{1}, \tau_{2}, \tau_{1}^{\prime}, \tau_{2}^{\prime}\right)$.

Proof. (1) First, we show that $\ell-\operatorname{dim}(X \times \mathbb{R}) \leq k$. For this, we represent $X \times \mathbb{R}=Z_{1} \cup Z_{2}$, where $Z_{1}=X \times \bigcup_{k \in \mathbb{Z}}(k, k+3 / 4), Z_{2}=X \times \bigcup_{k \in \mathbb{Z}}(k-1 / 2, k+1 / 4)$. Taking two appropriate qualified coverings of these sets and applying Proposition 3.2, we see that $\ell-\operatorname{dim}(X \times \mathbb{R}) \leq k$. Then taking an appropriate covering of $X \times \mathbb{R}$ and applying a homothety in the $\mathbb{R}$-factor, we find a desired covering.

(2) Consider the 2-colored covering $\mathcal{U}=\mathcal{U}^{1} \cup \mathcal{U}^{2}$ of $\mathbb{R}$, where $\mathcal{U}^{1}=\{(2 k, 2 k+2)$ : $k \in \mathbb{Z}\}, \mathcal{U}^{2}=\{(2 k+1,2 k+3): k \in \mathbb{Z}\}$. Clearly, $L(\mathcal{U})=1 / 2, \operatorname{mesh}(\mathcal{U})=2$.

There is a constant $\delta \in(0,1)$ such that for every sufficiently small $\tau_{1}, \tau_{2}>0$ there exists a $(k+1)$-colored open covering $\mathcal{V}$ of $X \times Y$ with $\operatorname{mesh}{ }^{\times}(\mathcal{V}) \leq\left(\tau_{1}, \tau_{2}\right)$ and $L^{\times}(\mathcal{V}) \geq$ $\delta\left(\tau_{1}, \tau_{2}\right)$.

By Corollary 3.6, for the product covering $\mathcal{W}=(\mathcal{V} * \mathcal{U}) * \mathcal{U}$ of $Z$ we have $L^{\times}(\mathcal{W}) \geq$ $q\left(\tau_{1}, \tau_{2}, 1 / 2,1 / 2\right)$ and $\operatorname{mesh}^{\times}(\mathcal{W}) \leq\left(\tau_{1}, \tau_{2}, 2,2\right)$, with some constant $q>0$ depending only on $k, \delta$. Applying homotheties independently in two $\mathbb{R}$-factors and changing the constants, we obtain a desired covering.

3.3. Locally self-similar spaces. Let $\lambda \geq 1$ and $R>0$ be given. A map $f: Z \rightarrow Z^{\prime}$ between metric spaces is $\lambda$-quasihomothetic with coefficient $R$ if for all $z, z^{\prime} \in Z$ we have

$$
R\left|z z^{\prime}\right| / \lambda \leq\left|f(z) f\left(z^{\prime}\right)\right| \leq \lambda R\left|z z^{\prime}\right| .
$$

A metric space $Z$ is locally self-similar if there is $\lambda \geq 1$ such that for every sufficiently large $R>1$ and every $A \subset Z$ with $\operatorname{diam} A \leq 1 / R$, there is a $\lambda$-quasihomothetic map $f: A \rightarrow Z$ with coefficient $R$.

In $[\mathrm{BL}]$ it was proved that the linearly controlled $\operatorname{dimension} \ell-\operatorname{dim} Z$ of every compact locally self-similar metric space $Z$ is finite and coincides with $\operatorname{dim} Z, \ell$-dim $Z=\operatorname{dim} Z$.

We shall use the following facts, obviously implied by the definition of a quasihomothetic map.

Lemma 3.8. Let $h: Z \rightarrow Z^{\prime}$ be a $\lambda$-quasihomothetic map with coefficient $R$. Let $V \subset Z$ and let $\widetilde{\mathcal{U}}$ be an open covering of $h(V), \mathcal{U}=h^{-1}(\widetilde{\mathcal{U}})$. Then:

(1) $R \operatorname{mesh}(\mathcal{U}) / \lambda \leq \operatorname{mesh}(\widetilde{\mathcal{U}}) \leq \lambda R \operatorname{mesh}(\mathcal{U})$;

(2) $\lambda R \cdot L(\mathcal{U}) \geq L(\widetilde{\mathcal{U}}) \geq R \cdot L(\mathcal{U}) / \lambda$, where $L(\mathcal{U})$ is the Lebesgue number of $\mathcal{U}$ as a covering of $V$.

Proposition 3.9. If compact metric spaces $X, Y$ are locally self-similar, then for the spaces of the family $a X \times b Y$ we have $\ell-\operatorname{dim} \leq n, n=\operatorname{dim}(X \times Y)$, uniformly in $a, b \geq 1$.

Proof. By the remark above, $\ell-\operatorname{dim} X=N$ is finite. Furthermore, the space $Z=X \times Y$ is compact and locally self-similar. Thus, $\ell-\operatorname{dim} Z=n$ is finite.

It suffices to prove that there is a constant $\delta \in(0,1)$ such that for every sufficiently small $\tau>0$ and every $\alpha, \beta \in(0,1]$ there exist $(n+1)$-colored open coverings $\mathcal{U}, \mathcal{U}^{\prime}$ of $Z$ with $\operatorname{mesh}^{\times}(\mathcal{U}) \leq(\alpha \tau, \tau), L^{\times}(\mathcal{U}) \geq \delta(\alpha \tau, \tau), \operatorname{mesh}^{\times}\left(\mathcal{U}^{\prime}\right) \leq(\tau, \beta \tau)$, and $L^{\times}\left(\mathcal{U}^{\prime}\right) \geq$ $\delta(\tau, \beta \tau)$.

Let $\delta_{1}, \delta_{2}, \delta_{3}$ be the constants occurring in the definition of $\ell$-dim for $X, Y, Z$, respectively, and let $\delta^{\prime}=\min \left\{\delta_{1}, \delta_{2}, \delta_{3}\right\}$. We may assume that the constant $\lambda$ in the definition of self-similarity serves both $X$ and $Y$. 
We explain how to construct the covering $\mathcal{U}$; the covering $\mathcal{U}^{\prime}$ is constructed similarly. Fix a positive $\tau<\min \left\{\delta^{\prime} / \lambda, \operatorname{diam} Y\right\}$. For every $\alpha \in(0,1]$, we construct a covering with mesh $^{\times} \leq(\alpha \tau, \tau)$ and $L^{\times} \geq \delta(\alpha \tau, \tau)$, where $\delta=\left(\delta^{\prime} / 4 \lambda^{2}\right)^{N+1} / 2$.

The argument is similar to that in [BL, Theorem 1.1]. We fix an $(N+1)$-colored open covering $\mathcal{V}^{\prime \prime}$ of $X$ with $\operatorname{mesh}\left(\mathcal{V}^{\prime \prime}\right) \leq \alpha / \lambda, L\left(\mathcal{V}^{\prime \prime}\right) \geq \delta^{\prime} \alpha / \lambda$ and put $\mathcal{V}=\left\{V \times Y: V \in \mathcal{V}^{\prime \prime}\right\}$. Then $\mathcal{V}$ is an $(N+1)$-colored open covering of $Z$ with $L(\mathcal{V}) \geq \delta^{\prime} \alpha / \lambda$, and we assume that $\mathcal{V}$ is colored by the set $S=\{0, \ldots, N\}, \mathcal{V}=\bigcup_{a \in S} \mathcal{V}^{a}$.

For every $V \in \mathcal{V}$, consider the slightly smaller subset $V^{\prime}=B_{-\delta^{\prime} \alpha / 2 \lambda}(V)$. Then, the sets $Z_{a}=\bigcup_{V \in \mathcal{V}^{a}} V^{\prime} \subset Z, a \in S$, cover $Z, Z=\bigcup_{a \in S} Z_{a}$, because $L(\mathcal{V}) \geq \delta^{\prime} \alpha / \lambda$. The idea is to construct a family of open $(n+1)$-colored coverings $\mathcal{U}_{a}$ of $Z_{a}, a \in S$, which is separated with and qualified by the scale function $i: S \rightarrow\{0, \ldots, N\}, i(a)=N-a$, and then to construct the desired $\mathcal{U}$ with the help of Proposition 3.2. The construction of $\mathcal{U}_{a}$ is based on the self-similarity of $X$.

Using the fact that $\ell-\operatorname{dim}(X \times Y)=n$ and assuming that $\tau$ is sufficiently small, for every $a \in S$ we can find an $(n+1)$-colored covering $\widetilde{\mathcal{U}}_{a}$ of $X \times Y$ with $\operatorname{mesh}\left(\widetilde{\mathcal{U}}_{a}\right) \leq$ $\left(\delta^{\prime} / 4 \lambda^{2}\right)^{a} \tau / 4$ and $L\left(\widetilde{\mathcal{U}}_{a}\right) \geq \delta^{\prime}\left(\delta^{\prime} / 4 \lambda^{2}\right)^{a} \tau / 4$.

Given $V \in \mathcal{V}$, we fix a map $h_{V}=\left(h_{V}^{1}\right.$, id $): V \rightarrow X \times Y$, where $h_{V}^{1}$ is $\lambda$-quasihomothetic with coefficient $R=\lambda / \alpha$, and put $\widetilde{V}=h_{V}\left(V^{\prime}\right)$. Now, for every $a \in S, V \in \mathcal{V}^{a}$, consider the family $\widetilde{\mathcal{U}}_{a, V}=\left\{\widetilde{U} \in \widetilde{\mathcal{U}}_{a}: \widetilde{V} \cap \widetilde{U} \neq \varnothing\right\}$, which is obviously an $(n+1)$-colored covering of $\widetilde{V}$. Then

$$
\mathcal{U}_{a, V}=\left\{h_{V}^{-1}(\widetilde{U}): \widetilde{U} \in \widetilde{\mathcal{U}}_{a, V}\right\}
$$

is an open $(n+1)$-colored covering of $V^{\prime}$.

Note that $U=h_{V}^{-1}(\widetilde{U}) \subset V$ for every $\widetilde{U} \in \widetilde{\mathcal{U}}_{a, V}$, because mesh ${ }^{\times} \widetilde{U} \leq(\tau / 4, \tau / 4)$. Therefore, $\operatorname{mesh}^{\times} U \leq(\alpha \tau / 4, \tau / 4)$ by Lemma 3.8, whence $U \subset B_{(\alpha \tau / 2, \operatorname{diam} Y)}\left(V^{\prime}\right) \subset V$ by the choice of $\tau$. Thus, the family $\mathcal{U}_{a, V}$ is contained in $V$. Now, the family $\mathcal{U}_{a}=$ $\bigcup_{V \in \mathcal{V}^{a}} \mathcal{U}_{a, V}$ covers the set $Z_{a}$ of the color $a$, and it has the following properties:

(1) for every $a \in S$, the covering $\mathcal{U}_{a}$ is $(n+1)$-colored;

(2) $L\left(\mathcal{U}_{a}\right)^{\bigotimes} \geq 4$ mesh $^{\bigotimes} \mathcal{U}_{a+1}$ for every $a \in S, a \leq N-1$;

(3) $\operatorname{mesh}^{\times}\left(\bigcup_{a \in S} \mathcal{U}_{a}\right) \leq(\alpha \tau, \tau), L^{\times}\left(\bigcup_{a \in S} \mathcal{U}_{a}\right) \geq\left(\delta^{\prime} / 4 \lambda^{2}\right)^{N+1}(\alpha \tau, \tau)$.

Indeed, since distinct $V_{1}, V_{2} \in \mathcal{V}^{a}$ are disjoint, any $U_{1} \in \mathcal{U}_{a, V_{1}}, U_{2} \in \mathcal{U}_{a, V_{2}}$ are disjoint because $U_{1} \subset V_{1}, U_{2} \subset V_{2}$. This proves (1).

Lemma 3.8 shows that $\operatorname{mesh}^{\times}\left(\mathcal{U}_{a}\right) \leq \operatorname{mesh}\left(\widetilde{\mathcal{U}}_{a}\right)(\alpha, 1)$ and $L^{\times}\left(\mathcal{U}_{a}\right) \geq L\left(\widetilde{\mathcal{U}}_{a}\right)\left(\alpha / \lambda^{2}, 1 / \lambda^{2}\right)$ for every $a \in S$. These estimates together with the estimates on mesh $\left(\widetilde{\mathcal{U}}_{a}\right)$ and $L\left(\widetilde{\mathcal{U}}_{a}\right)$ yield (3), and together with the inequalities

$$
4 \lambda^{2} \operatorname{mesh}\left(\widetilde{\mathcal{U}}_{a+1}\right) \leq \delta^{\prime}\left(\delta^{\prime} / 4 \lambda^{2}\right)^{a} \tau / 4 \leq L\left(\widetilde{\mathcal{U}}_{a}\right)
$$

for every $a \in S, a \leq N-1$, yield (2).

In view of (1) and (2), the family of coverings $\mathcal{U}_{a}, a \in S$, satisfies the assumption of Proposition 3.2. Applying that proposition and using (3), we obtain an open $(n+1)$ colored covering $\mathcal{U}$ of $X \times Y$ with $\operatorname{mesh}^{\times}(\mathcal{U}) \leq(\alpha \tau, \tau)$ and $L^{\times}(\mathcal{U}) \geq \delta(\alpha \tau, \tau)$, where $\delta=\left(\delta^{\prime} / 4 \lambda^{2}\right)^{N+1} / 2$.

3.4. "Hyperbolic cone" map. Let $Z$ be a bounded metric space. Assuming that $\operatorname{diam} Z>0$, we put $\mu=\pi / \operatorname{diam} Z$ and note that $\mu\left|z z^{\prime}\right| \in[0, \pi]$ for every $z, z^{\prime} \in Z$. Recall that the hyperbolic cone $\operatorname{Co}(Z)$ over $Z$ is the space $Z \times[0, \infty) / Z \times\{0\}$ with metric defined as follows. Given $x=(z, t), x^{\prime}=\left(z^{\prime}, t^{\prime}\right) \in \operatorname{Co}(Z)$, we consider a triangle $\bar{o} \bar{x} \bar{x}^{\prime} \subset \mathrm{H}^{2}$ with $|\bar{o} \bar{x}|=t,\left|\bar{o} \bar{x}^{\prime}\right|=t^{\prime}$, and the angle $\angle_{\bar{o}}\left(\bar{x}, \bar{x}^{\prime}\right)=\mu\left|z z^{\prime}\right|$. Now, we put $\left|x x^{\prime}\right|:=\left|\bar{x} \bar{x}^{\prime}\right|$. In the degenerate case $Z=\{\mathrm{pt}\}$, we define $\operatorname{Co}(Z)=\{\mathrm{pt}\} \times[0, \infty)$ as the metric product. The point $o=Z \times\{0\} \in \operatorname{Co}(Z)$ is called the vertex of $\operatorname{Co}(Z)$. 
We let $h: Z \times[0, \infty) \rightarrow \mathrm{Co}(Z)$ be the canonical projection, and $\delta=\delta_{\mathrm{H}^{2}}$ the hyperbolicity constant of $\mathrm{H}^{2}$.

It is well known that the hyperbolic cone $\operatorname{Co}(Z)$ is a hyperbolic space satisfying the $\delta$-inequality with respect to the vertex $o$. Furthermore, there is a canonical inclusion $Z \subset \partial_{\infty} \operatorname{Co}(Z)$, and the metric of $Z$ is visual with respect to the base point $o$ and the parameter $e$,

$$
e^{-\left(\xi \mid \xi^{\prime}\right)_{o}-c_{0}} \leq d\left(\xi, \xi^{\prime}\right) \leq e^{-\left(\xi \mid \xi^{\prime}\right)_{o}+c_{0}}
$$

for some $c_{0} \geq 0$ and all $\xi, \xi^{\prime} \in Z$. In general, $\operatorname{Co}(Z)$ is not geodesic; however, for every point $z \in \operatorname{Co}(Z)$ there is a geodesic segment $o z \subset \operatorname{Co}(Z)$, and every $\xi \in Z$ is represented by a geodesic ray $o \xi \subset \mathrm{Co}(Z)$.

The following lemma is similar to [BoS, Lemma 5.1].

Lemma 3.10. Let $X$ be a $\delta$-hyperbolic space. Given o, $z_{1}, z_{2} \in X$ and $x_{1} \in o z_{1}$, $x_{2} \in o z_{2}$, we have

$$
\min \left\{\left|o x_{1}\right|,\left|o x_{2}\right|,\left(z_{1} \mid z_{2}\right)_{o}\right\}-2 \delta \leq\left(x_{1} \mid x_{2}\right)_{o} \leq \min \left\{\left|o x_{1}\right|,\left|o x_{2}\right|,\left(z_{1} \mid z_{2}\right)_{o}\right\}+2 \delta .
$$

Proof. Applying the $\delta$-inequality twice, we obtain

$$
\begin{aligned}
\left(z_{1} \mid z_{2}\right)_{o} & \geq \min \left\{\left(z_{1} \mid x_{1}\right)_{o},\left(x_{1} \mid x_{2}\right)_{o},\left(x_{2} \mid z_{2}\right)_{o}\right\}-2 \delta \\
& =\min \left\{\left|o x_{1}\right|,\left(x_{1} \mid x_{2}\right)_{o},\left|o x_{2}\right|\right\}-2 \delta=\left(x_{1} \mid x_{2}\right)_{o}-2 \delta .
\end{aligned}
$$

Similarly, $\left(x_{1} \mid x_{2}\right)_{o} \geq \min \left\{\left|o x_{1}\right|,\left(z_{1} \mid z_{2}\right)_{o},\left|o x_{2}\right|\right\}-2 \delta$. The lemma follows.

Corollary 3.11. Let $X$ be a $\delta$-hyperbolic space. Suppose that points $x_{1} \in o \xi_{1}, x_{2} \in o \xi_{2}$ satisfy $\left|x_{1} o\right|=\left|x_{2} o\right|$ and $\left|x_{1} x_{2}\right|>4 \delta$, where $\xi_{1}, \xi_{2} \in \partial_{\infty} X$. Then

$$
\left(x_{1} \mid x_{2}\right)_{o}-2 \delta \leq\left(\xi_{1} \mid \xi_{2}\right)_{o} \leq\left(x_{1} \mid x_{2}\right)_{o}+2 \delta .
$$

Proof. In this case $\left(x_{1} \mid x_{2}\right)_{o}<\left|o x_{1}\right|-2 \delta=\left|o x_{2}\right|-2 \delta$. Hence, by the preceding lemma, $\min \left\{\left|o x_{1}\right|,\left|o x_{2}\right|,\left(z_{1} \mid z_{2}\right)_{o}\right\}=\left(z_{1} \mid z_{2}\right)_{o}$ for each $z_{1} \in\left(x_{1}, \xi_{1}\right), z_{2} \in\left(x_{2}, \xi_{2}\right)$. Now, the statement follows from that lemma.

We put $c=c_{0}+2 \delta$.

Claim 1. For every $R>0$, the map $\left.h\right|_{Z \times\{R\}}: Z \times\{R\} \rightarrow \operatorname{Co}(Z)$ possesses the following properties:

(1) $\left|h(z, R) h\left(z^{\prime}, R\right)\right| \geq 2 D$ for every $D>0$ and every $z, z^{\prime} \in Z$ with $\left|z z^{\prime}\right| \geq e^{-R+D+c}$;

(2) $\left|h(z, R) h\left(z^{\prime}, R\right)\right| \leq 2 D$ for every $D>2 \delta$ and every $z$, $z^{\prime} \in Z$ with $\left|z z^{\prime}\right| \leq$ $e^{-R+D-c}$.

Proof. (1) We identify $z=\xi, z^{\prime}=\xi^{\prime} \in \partial_{\infty} \operatorname{Co}(Z)$ and denote $h(z, R)=x, h\left(z^{\prime}, R\right)=x^{\prime}$. Thus,

$$
e^{-R+D+c} \leq\left|\xi \xi^{\prime}\right| \leq e^{-\left(\xi \mid \xi^{\prime}\right)_{o}+c_{0}},
$$

and we obtain $\left(\xi \mid \xi^{\prime}\right)_{o} \leq R-D-2 \delta$. By Lemma 3.10,

$$
\left(x \mid x^{\prime}\right)_{o} \leq \min \left\{R,(\xi \mid \xi)_{o}\right\}+2 \delta \leq R-D .
$$

Consequently, $\left|x x^{\prime}\right|=2\left(R-\left(x \mid x^{\prime}\right)_{o}\right) \geq 2 D$.

(2) Using the notation as above, we obtain $e^{-\left(\xi \mid \xi^{\prime}\right)_{o}-c_{0}} \leq\left|\xi \xi^{\prime}\right| \leq e^{-R+D-c}$. Hence, $\left(\xi \mid \xi^{\prime}\right)_{o} \geq R-D+2 \delta$. In the case where $\left|x x^{\prime}\right| \leq 4 \delta$, we see that $\left|x x^{\prime}\right|<2 D$ by the assumption $D>2 \delta$. If $\left|x x^{\prime}\right|>4 \delta$, we apply Corollary 3.11, obtaining $\left(x \mid x^{\prime}\right)_{o} \geq\left(\xi \mid \xi^{\prime}\right)_{o}-$ $2 \delta \geq R-D$. Thus, $\left|x x^{\prime}\right|=2\left(R-\left(x \mid x^{\prime}\right)_{o}\right) \leq 2 D$.

The proof of the following claim is similar, and we leave it to the reader as an exercise. We only mention that at some point one should use the monotonicity of the Gromov product, $\left(x \mid x^{\prime}\right)_{o} \leq\left(\xi \mid \xi^{\prime}\right)_{o}$ for every $x \in o \xi, x^{\prime} \in o \xi^{\prime}$. 
Claim 2. For every $R>0$, the map $\left.h\right|_{Z \times\{R\}}: Z \times\{R\} \rightarrow \operatorname{Co}(Z)$ possesses the following properties:

(1) $\left|z z^{\prime}\right| \geq e^{-R+D-c}$ for every $D>2 \delta$ and every $x=h(z, R), x^{\prime}=h\left(z^{\prime}, R\right)$ with $\left|x x^{\prime}\right| \geq 2 D$

(2) $\left|z z^{\prime}\right| \leq e^{-R+D+c}$ for every $D>0$ and every $x=h(z, R), x^{\prime}=h\left(z^{\prime}, R\right)$ with $\left|x x^{\prime}\right| \leq 2 D$.

Claim 3. (1) Given $l>0, R \geq R_{1} \geq l$, put $r=e^{-R_{1}+3 l / 2+c}$. Then

$$
h\left(B_{r}(x) \times[R-l, R+l]\right) \supset B_{l}(h(x, R)) .
$$

(2) Given $l>2 \delta, t>0, R_{2} \geq R \geq t$, put $r=e^{-R_{2}+l-c}$. Then

$$
h\left(B_{r}(x) \times[R-t, R+t]\right) \subset B_{3 t+2 l}(h(x, R)) .
$$

Proof. (1) Given $y^{\prime} \in \mathrm{Co}(Z)$ with $\left|y^{\prime} h(x, R)\right|<l$, we represent $y^{\prime}=h\left(y, R_{0}\right)$. Using the definition of the distances in $\mathrm{Co}(Z)$, we see that $R_{0} \in[R-l, R+l]$ and $\left|h\left(y, R^{\prime}\right) h\left(x, R^{\prime}\right)\right|<$ $l$, where $R^{\prime}=\min \left\{R_{0}, R\right\}$. Then, by Claim 2(2), we have

$$
|y x| \leq e^{-R^{\prime}+l / 2+c} \leq e^{-R+l+l / 2+c} \leq e^{-R_{1}+3 l / 2+c} .
$$

Claim 3(1) follows.

(2) Assume that $\left(y, R_{0}\right) \in B_{r}(x) \times[R-t, R+t]$. Then $R_{0} \in[R-t, R+t]$ and $|y x|<e^{-R_{2}+l-c} \leq e^{-R_{0}+t+l-c}$. By Claim $1(2)$, we have $\left|h\left(y, R_{0}\right) h\left(x, R_{0}\right)\right|<2 t+2 l$. By the triangle inequality,

Claim 3(2) follows.

$$
\left|h(x, R) h\left(y, R_{0}\right)\right|<3 t+2 l .
$$

Claim 4. (1) Given $R_{1} \geq R>0, l>8 \delta$, put $r_{1}=e^{-R_{1}-l / 4-c}$. Then $h^{-1}\left(B_{l}(h(x, R))\right) \supset$ $B_{r_{1}}(x) \times[R-l / 2, R+l / 2]$.

(2) Given $l>0, R \geq R_{2} \geq l$, put $r_{2}=e^{-R_{2}+3 l / 2+c}$. Then $h^{-1}\left(B_{l}(h(x, R))\right) \subset$ $B_{r_{2}}(x) \times[R-l, R+l]$.

Proof. (1) Assume that $\left(y, R_{0}\right) \in B_{r_{1}}(x) \times[R-l / 2, R+l / 2]$. Then $R_{0} \in[R-l / 2, R+l / 2]$ and $|x y|<e^{-R_{1}-l / 4-c} \leq e^{-R_{0}+l / 4-c}$. Using Claim 1(2), we obtain $\left|h\left(y, R_{0}\right) h\left(x, R_{0}\right)\right|<$ $l / 2$. Then $\left|h\left(y, R_{0}\right) h(x, R)\right|<l$ by the triangle inequality. Claim 4(1) follows.

(2) Claim 4(2) follows from Claim 3(1).

\section{§4. ProOFs}

Proposition 4.1. Let $Z_{1}, Z_{2}$ be bounded metric spaces such that for the spaces of the family $\left\{a Z_{1} \times b Z_{2}: a \geq 1, b \geq 1\right\}$ we have $\ell$-dim $\leq k$ uniformly. Then

$$
\operatorname{asdim}\left(\mathrm{Co}\left(Z_{1}\right) \times \operatorname{Co}\left(Z_{2}\right)\right) \leq k+2 .
$$

We need some preparation for the proof. We denote

$$
\begin{aligned}
& \overline{P_{0}}(T)=Z_{1} \times[0, T] \times Z_{2} \times[0, T], \\
& \overline{P_{1}}(T)=Z_{1} \times[T, \infty] \times Z_{2} \times[0, T], \\
& \overline{P_{2}}(T)=Z_{1} \times[0, T] \times Z_{2} \times[T, \infty], \\
& \overline{P_{3}}(T)=Z_{1} \times[T, \infty] \times Z_{2} \times[T, \infty],
\end{aligned}
$$

and $P_{i}=\left(h_{1}, h_{2}\right)\left(\overline{P_{i}}\right)$, where $h_{i}: Z_{i} \times[0, \infty) \rightarrow$ Co $Z_{i}$ is the canonical projection, $i=1,2$.

Lemma 4.2. For every $L>0$ there exists $T_{3}$ and $M>0$ such that for every $T>T_{3}$ there exists an $(L, M, k+3)$-covering of $P_{3}(T)$. 
Proof. Let $\sigma>1$ be the constant in Lemma 3.7(2). We fix a constant $L>0$, put $H=512 \sigma^{4} L$, and for any integers $m, n \geq 0$ consider the product

$$
A_{m, n}=Z_{1} \times Z_{2} \times[H m, H m+H] \times[H n, H n+H] .
$$

Fixing some $T>0$, first we construct a $(k+3)$-colored covering $\mathcal{U}$ of $\overline{P_{3}}(T)$ such that for all sufficiently large integers $m, n$ we have

$$
\begin{gathered}
\left.L^{\times}(\mathcal{U})\right|_{A_{m, n}} \geq\left(e^{-H m+3 L / 2+c}, e^{-H n+3 L / 2+c}, L, L\right), \\
\left.\operatorname{mesh}^{\times}(\mathcal{U})\right|_{A_{m, n}} \leq\left(e^{D} e^{-H m}, e^{D} e^{-H n}, H / 4, H / 4\right)
\end{gathered}
$$

for some $D>0$, where $c>0$ is the constant defined before Claim 1 .

It is convenient to denote $c_{1}=2 e^{3 L / 2+c}, b=e^{-H}$. Using Lemma 3.7(2), we fix $N$ so large that for all integers $m, n \geq N$ and every $i=0,1,2,3$ there exists a $(k+3)$-colored covering $\widetilde{\mathcal{U}}_{m, n}^{i}$ of $Z_{1} \times Z_{2} \times \mathbb{R} \times \mathbb{R}$ with $L^{\times}\left(\widetilde{\mathcal{U}}_{m, n}^{i}\right) \geq(4 \sigma)^{i}\left(c_{1} b^{m-i}, c_{1} b^{n-i}, 2 L, 2 L\right)$ and $\operatorname{mesh}^{\times}\left(\widetilde{\mathcal{U}}_{m, n}^{i}\right) \leq \sigma(4 \sigma)^{i}\left(c_{1} b^{m-i}, c_{1} b^{n-i}, 2 L, 2 L\right)$. For $m, n \geq N$, we consider the subfamily $\mathcal{U}_{m, n}^{i}$ of $\widetilde{\mathcal{U}}_{m, n}^{i}$ consisting of all members that intersect $A_{m, n}$.

Next, we define a function $i: \mathbb{N} \times \mathbb{N} \rightarrow\{0,1,2,3\}$ by $i(m, n):=m \bmod 2+$ $2(n \bmod 2)$ and put $\mathcal{U}_{m, n}:=\mathcal{U}_{m, n}^{i(m, n)}$. We show that the family of coverings $\mathcal{U}_{m, n}$ of $A_{m, n}, m, n \geq N$, is separated with and qualified by the scale function $i$.

We have $\sigma(4 \sigma)^{3} 2 L=H / 4<H / 2$ by the choice of the constants. The estimate on mesh $^{\times}\left(\widetilde{\mathcal{U}}_{m, n}^{i}\right)$ implies that any member $U \in \mathcal{U}_{m, n}$ is disjoint with any $U^{\prime} \in \mathcal{U}_{m^{\prime}, n^{\prime}}$ if $i(m, n)=i\left(m^{\prime}, n^{\prime}\right)$ and $(m, n) \neq\left(m^{\prime}, n^{\prime}\right)$. That is, the family $\mathcal{U}_{m, n}, m, n \geq N$, is separated with the scale function $i$. In particular, if $U \cap U^{\prime} \neq \varnothing$ for some $U \in \mathcal{U}_{m, n}$, $U^{\prime} \in \mathcal{U}_{m^{\prime}, n^{\prime}}$, then the pairs $(m, n)$ and $\left(m^{\prime}, n^{\prime}\right)$ are adjacent; i.e., $\left|m^{\prime}-m\right| \leq 1$ and $\left|n^{\prime}-n\right| \leq 1$.

Assume that the pairs $\left(m^{\prime}, n^{\prime}\right),(m, n)$ are adjacent and $i\left(m^{\prime}, n^{\prime}\right)>i(m, n)$. We have $(4 \sigma)^{i+1} 2 L=4 \sigma(4 \sigma)^{i} 2 L$ and $(4 \sigma)^{i+1} c_{1} b^{m-(i+1)}>4 \sigma(4 \sigma)^{i} c_{1} b^{m-i}$, because $b<1$. These inequalities together with the estimates on $L^{\times}\left(\widetilde{\mathcal{U}}_{m, n}^{i}\right)$ and $\operatorname{mesh}^{\times}\left(\widetilde{\mathcal{U}}_{m, n}^{i}\right)$ show that

$$
L^{\bigotimes}\left(\mathcal{U}_{m^{\prime}, n^{\prime}}\right) \geq 4 \operatorname{mesh}^{\bigotimes}\left(\mathcal{U}_{m, n}\right) .
$$

It follows that these coverings are qualified by the scale function $i$. Applying Proposition 3.2 to this family of coverings, we obtain a $(k+3)$-colored covering $\mathcal{U}$ of $\bar{P}_{3}(T)$, $T=H N$, for which

$$
\begin{aligned}
\left.L^{\times}(\mathcal{U})\right|_{A_{m, n}} & \geq \frac{1}{2}\left(c_{1} e^{-H m}, c_{1} e^{-H n}, 2 L, 2 L\right) \\
& =\left(e^{-H m+3 L / 2+c}, e^{-H n+3 L / 2+c}, L, L\right), \\
\left.\operatorname{mesh}^{\times}(\mathcal{U})\right|_{A_{m, n}} & \leq \sigma(4 \sigma)^{3}\left(c_{1} e^{-H(m-i(m, n))}, c_{1} e^{-H(n-i(m, n))}, 2 L, 2 L\right) \\
& \leq\left(e^{D} e^{-H m}, e^{D} e^{-H n}, H / 4, H / 4\right)
\end{aligned}
$$

for some $D>0$.

Now, we estimate the Lebesgue number and the mesh of the covering $\mathcal{U}^{\prime}=\left(h_{1}, h_{2}\right)(\mathcal{U})$. Applying Claim 3(1) with $l=L, R_{1}=H_{m}, R \in[H m, H m+H]$ (respectively, $R_{1}=H n$, $R \in[H n, H n+H]$ ) to the map $h_{1}$ (respectively, $h_{2}$ ), we obtain $L\left(\mathcal{U}^{\prime}\right) \geq L$.

Next, we represent the upper estimate for $\left.\operatorname{mesh}^{\times}(\mathcal{U})\right|_{A_{m, n}}$ as follows:

$$
\left.\operatorname{mesh}^{\times}(\mathcal{U})\right|_{A_{m, n}} \leq\left(e^{-(H m+H)+H+D+c-c}, e^{-(H n+H)+H+D+c-c}, H / 4, H / 4\right) .
$$

Now, applying Claim 3(2) with $l=H+D+c$ (note that $\left.l>2 \delta=2 \delta_{\mathrm{H}^{2}}\right), t=H / 4$, $R_{2}=H m+H, R \in[H m, H m+H]$ (respectively, $R_{2}=H n+H, R \in[H n, H n+H]$ ) to the map $h_{1}$ (respectively, $h_{2}$ ), we obtain $\operatorname{mesh}\left(\mathcal{U}^{\prime}\right) \leq M=2(3 H / 4+2(H+D+c)$ ).

It follows that $\mathcal{U}^{\prime}$ is an $(L, M, k+3)$-covering of $P_{3}(T)$. 
Lemma 4.3. Given $L>0$, there is $T_{2}$ such that for every $T>T_{2}$ there exist $(L, M, k+2)$ coverings of $P_{1}(T), P_{2}(T)$ with $M>0$ depending on $T, L$.

Proof. The proof is similar to that of Lemma 4.2. For simplicity, we construct a desired covering of $P_{1}(T)$. For $P_{2}(T)$ the construction is the same with obvious modifications.

We fix $L>0$ and put $H=40 \sigma^{2} L$. Let $\sigma$ be the constant in Lemma 3.7(1). For every integer $m \geq 0$, consider the product

$$
A_{m}=Z_{1} \times Z_{2} \times[H m, H m+H] \times[0, T] .
$$

For some $T>0$, first we construct a $(k+2)$-colored covering $\mathcal{U}$ of $\overline{P_{1}}(T)$ such that for all $m \geq N$ we have

$$
\begin{gathered}
\left.L(\mathcal{U})\right|_{A_{m}} \geq\left(e^{-H m+3 L / 2+c}, \operatorname{diam} Z_{2}, L, L\right), \\
\left.\operatorname{mesh}(\mathcal{U})\right|_{A_{m}} \leq\left(e^{D} e^{-H m}, \operatorname{diam} Z_{2}, H / 4, T+L\right)
\end{gathered}
$$

for some $D>0$, where $c=c_{0}+2 \delta$ is the constant introduced before Claim 1 .

For convenience, we put $c_{1}=2 e^{3 L / 2+c}, b=e^{-H}$. Since $Z_{1}$ is isometrically embedded into $Z_{1} \times Z_{2}$, we have $\ell$ - $\operatorname{dim} Z_{1} \leq \ell-\operatorname{dim}\left(Z_{1} \times Z_{2}\right) \leq k$, whence $\ell-\operatorname{dim}\left(Z_{1} \times[0,1]\right) \leq k+1$. Then, using Lemma 3.7(1), we can find $N$ so large that for every integer $m \geq N$ and every $i=0,1$ there exists a $(k+2)$-colored covering $\widetilde{\mathcal{U}}_{m}^{i}$ of $Z_{1} \times \mathbb{R}$ with

$$
\begin{aligned}
L^{\times}\left(\tilde{\mathcal{U}}_{m}^{i}\right) & \geq(4 \sigma)^{i}\left(c_{1} b^{m-i}, 2 L\right), \\
\operatorname{mesh}^{\times}\left(\tilde{\mathcal{U}}_{m}^{i}\right) & \leq \sigma(4 \sigma)^{i}\left(c_{1} b^{m-i}, 2 L\right) .
\end{aligned}
$$

We take its subfamily $\mathcal{U}_{m}^{i}$ consisting of all members that intersect $\widehat{A}_{m}=Z_{1} \times[H m$, $H m+h]$.

We define a function $i: \mathbb{N} \rightarrow\{0,1\}$ by $i(m):=m \bmod 2$ and put $\mathcal{U}_{m}:=\mathcal{U}_{m}^{i(m)}$. Now, we show that the family of the coverings $\mathcal{U}_{m}$ of $\widehat{A}_{m}, m \geq N$, is separated with and qualified by the scale function $i$.

We have $\sigma(4 \sigma) 2 L<H / 4$ by the choice of the constants. The estimate on mesh ${ }^{\times}\left(\widetilde{\mathcal{U}}_{m}^{i}\right)$ implies that any member $U \in \mathcal{U}_{m}$ is disjoint with any $U^{\prime} \in \mathcal{U}_{m^{\prime}}$ if $i(m)=i\left(m^{\prime}\right)$ and $m \neq m^{\prime}$. That is, the family $\mathcal{U}_{m}, m \geq N$, is separated with the scale function $i$. In particular, if $U \cap U^{\prime} \neq \varnothing$ for some $U \in \mathcal{U}_{m}, U^{\prime} \in \mathcal{U}_{m^{\prime}}$, then $\left|m^{\prime}-m\right| \leq 1$.

Assume that $\left|m^{\prime}-m\right|=1$ and $i\left(m^{\prime}\right)>i(m)$; i.e., $i\left(m^{\prime}\right)=1, i(m)=0$. Using the estimates on $L^{\times}\left(\widetilde{\mathcal{U}}_{m}^{i}\right)$ and $\operatorname{mesh}^{\times}\left(\widetilde{\mathcal{U}}_{m}^{i}\right)$, and recalling that $b<1$, we obtain

$$
L^{\bigotimes}\left(\mathcal{U}_{m^{\prime}}\right) \geq 4 \operatorname{mesh}^{\otimes}\left(\mathcal{U}_{m}\right) .
$$

Thus, these coverings are qualified by the scale function $i$. So, we apply Proposition 3.2 to the family of the coverings $\mathcal{U}_{m}, m \geq N$, and obtain a $(k+2)$-colored covering $\mathcal{U}^{\prime}$ of $Z_{1} \times[T, \infty), T=H N$, with

$$
\begin{aligned}
\left.L^{\times}\left(\mathcal{U}^{\prime}\right)\right|_{A_{m}} & \geq \frac{1}{2}\left(c_{1} e^{-H m}, 2 L\right)=\left(e^{-H m+3 L / 2+c}, L\right), \\
\left.\operatorname{mesh}^{\times}\left(\mathcal{U}^{\prime}\right)\right|_{A_{m}} & \leq 4 \sigma^{2}\left(c_{1} e^{-H(m-i(m))}, 2 L\right) \leq\left(e^{D} e^{-H m}, H / 4\right)
\end{aligned}
$$

for some $D>0$.

Now, the family $\mathcal{U}=\left\{U \times Z_{2} \times[0, T+L]: U \in \mathcal{U}^{\prime}\right\}$ is a $(k+2)$-colored covering of $\overline{P_{1}}(T)$ that satisfies the required estimates on $L^{\times}$- and mesh ${ }^{\times}$-numbers.

We estimate the Lebesgue number and the mesh of the covering $\mathcal{U}^{\prime \prime}=\left(h_{1}, h_{2}\right)(\mathcal{U})$. Applying Claim 3(1) with $l=L, R_{1}=H_{m}$, and $R \in[H m, H m+H]$ to the map $h_{1}$, we obtain $L\left(\mathcal{U}^{\prime \prime}\right) \geq L$.

Next, we represent the upper estimate for $\left.\operatorname{mesh}^{\times}(\mathcal{U})\right|_{A_{m}}$ as follows:

$$
\left.\operatorname{mesh}^{\times}(\mathcal{U})\right|_{A_{m}} \leq\left(e^{-(H m+H)+H+D+c-c}, \operatorname{diam} Z_{2}, H / 4, T+L\right) .
$$


Applying Claim 3(2) with $l=H+D+c$ (note that $\left.l>2 \delta=2 \delta_{\mathrm{H}^{2}}\right), t=H / 4, R_{2}=$ $H m+2 H$, and $R \in[H m, H m+H]$ to the map $h_{1}$ and using the fact that the diameter of $h_{2}\left(Z_{2} \times[0, T+L]\right) \subset \mathrm{Co}\left(Z_{2}\right)$ is at most $2(T+L)$, we obtain $\operatorname{mesh}\left(\mathcal{U}^{\prime \prime}\right) \leq M=$ $2 \max \{3 H / 4+2(2 H+D+c), T+L\}$.

It follows that $\mathcal{U}^{\prime \prime}$ is an $(L, M, k+2)$-covering of $P_{1}(T)$.

Lemma 4.4. For every $T, L>0$ there exists an $(L, M, 1)$-covering of $P_{0}(T)$ with $M>0$ depending only on $T$ and $L$.

Proof. The covering of $P_{0}(T)$ consisting of a unique element $U=h_{1}\left(Z_{1} \times[0, T+2 L)\right) \times$ $h_{2}\left(Z_{2} \times[0, T+2 L)\right)$ has the required properties.

Proof of Proposition 4.1. Given $L_{0}>0$, we construct a uniformly bounded covering $\mathcal{U}$ of $\operatorname{Co}\left(Z_{1}\right) \times \operatorname{Co}\left(Z_{2}\right)$ with $L(\mathcal{U}) \geq L_{0}$.

We apply Lemma 4.2 with $L=L_{0}$, find the corresponding constants $T_{3}, M_{3}$, and let $\mathcal{U}_{3}$ be an $\left(L_{0}, M_{3}, k+3\right)$-covering of $P_{3}\left(T_{3}\right)$. We may assume that $M_{3} \geq L_{0}$.

Next, we apply Lemma 4.3 with $L=2 M_{3}$ and denote by $T_{2}$ the corresponding constant. Thus, for $T_{0}=\max \left\{T_{2}, T_{3}\right\}$ there is a $\left(2 M_{3}, M_{2}, k+2\right)$-covering $\mathcal{U}_{2}$ of $P_{2}\left(T_{0}\right)$, where the constant $M_{2} \geq 2 M_{3}$ depends on $T_{0}, M_{3}$.

Then we again apply Lemma 4.3, this time with $L=2 M_{2}$, and denote by $T_{1}$ the corresponding constant. Thus, for $T^{*}=\max \left\{T_{0}, T_{1}\right\}$ there is a $\left(2 M_{2}, M_{1}, k+2\right)$-covering $\mathcal{U}_{1}$ of $P_{1}\left(T^{*}\right)$, where the constant $M_{1} \geq 2 M_{2}$ depends on $T^{*}$ and $M_{2}$.

Finally, by Lemma 4.4 , there is a $\left(2 M_{1}, M, 1\right)$-covering $\mathcal{U}_{0}$ of $P_{0}\left(T^{*}\right)$, where $M>0$ depends on $M_{1}$ and $T^{*}$.

We have

$$
\operatorname{Co}\left(Z_{1}\right) \times \operatorname{Co}\left(Z_{2}\right)=P_{0}\left(T^{*}\right) \cup P_{1}\left(T^{*}\right) \cup P_{2}\left(T_{0}\right) \cup P_{3}\left(T_{3}\right) .
$$

Now we apply Lemma 3.1 consecutively to the sequence of the coverings $\mathcal{U}_{0}, \mathcal{U}_{1}, \mathcal{U}_{2}$, $\mathcal{U}_{3}$, obtaining a bounded $(k+3)$-colored covering $\mathcal{U}$ of $\operatorname{Co}\left(Z_{1}\right) \times \operatorname{Co}\left(Z_{2}\right)$ with $L(\mathcal{U}) \geq L_{0}$. This shows that $\operatorname{asdim}\left(\mathrm{Co}\left(Z_{1}\right) \times \operatorname{Co}\left(Z_{2}\right)\right) \leq k+2$.

Proposition 4.5. Let $Z$ be a bounded metric space. Then

$$
\ell \text {-asdim } \operatorname{Co}(Z) \leq \ell-\operatorname{dim}(Z \times[0,1]) .
$$

Proof. We may assume that $\ell-\operatorname{dim}(Z \times[0,1])=k$ is finite. We fix some $L>4 \delta, \delta=\delta_{\mathrm{H}^{2}}$. Let $\tau_{0}>0, \sigma>1$ be the constants in Lemma 3.7(1). We put $H=40 \sigma^{2} L$ and denote

$$
A_{m}=Z \times[H m, H m+H] .
$$

There exists $N>0$ such that for $m \geq N$ we have $e^{-H(m-1)+3 L / 2+c}<\tau_{0}$ uniformly in $L>4 \delta$, where, as above, $c=c_{0}+2 \delta$ is the constant introduced before Claim 1 .

Precisely as in the proof of Lemma 4.3 , we construct a $(k+1)$-colored covering $\mathcal{U}$ of $Z \times[H N, \infty)$ such that

$$
\begin{aligned}
\left.L^{\times}(\mathcal{U})\right|_{A_{m}} & \geq\left(e^{-H m+3 L / 2+c}, L\right), \\
\left.\operatorname{mesh}^{\times}(\mathcal{U})\right|_{A_{m}} & \leq\left(e^{D} e^{-H m}, H / 4\right)
\end{aligned}
$$

for all $m \geq N$, for some constant $D>0$ linearly depending on $L$.

We have

$$
\left.\operatorname{mesh}^{\times}(\mathcal{U})\right|_{A_{m}} \leq\left(e^{-(H m+H)+(H+3 L / 2+D+c)-c}, H / 4\right) .
$$

Applying Claim 3 to the canonical map $h: Z \times[0, \infty) \rightarrow \mathrm{Co}(Z)$, we see that the covering $h(\mathcal{U})=\mathcal{U}^{\prime}$ satisfies $L\left(\mathcal{U}^{\prime}\right) \geq L$ and $\operatorname{mesh}\left(\mathcal{U}^{\prime}\right) \leq(3 H / 4+2(H+D+3 L / 2+c))$.

Now for every $L>4 \delta$ we have the $(k+1)$-colored covering $\mathcal{U}^{\prime}$ of $h(Z \times[C L, \infty))$ with $L\left(\mathcal{U}^{\prime}\right) \geq L$ and $\operatorname{mesh}\left(\mathcal{U}^{\prime}\right) \leq C L$ for some constant $C \geq 1$ independent of $L$. Then we cover the ball $B_{C L}(o) \subset$ Co $Z$ by the ball $\mathcal{U}^{\prime \prime}=B_{3 C L}(o)$ and apply Lemma 3.1 to $\mathcal{U}^{\prime}$, 
$\mathcal{U}^{\prime \prime}$ to obtain a covering $\mathcal{W}$ of Co $Z$ with multiplicity $m(\mathcal{W}) \leq k+1$ and with $L(\mathcal{W}) \geq L$ and $\operatorname{mesh}(\mathcal{W}) \leq 6 C L$. This shows that $\operatorname{asdim} \operatorname{Co}(Z) \leq \ell-\operatorname{dim}(Z \times[0,1])$.

Proposition 4.6. Let $Z_{1}, \ldots, Z_{n}$ be bounded metric spaces. Then

$$
\ell-\operatorname{dim}\left(Z_{1} \times \cdots \times Z_{n} \times[0,1]^{n}\right) \leq \operatorname{asdim}\left(\operatorname{Co} Z_{1} \times \cdots \times \operatorname{Co} Z_{n}\right) .
$$

We reduce the proof to the following two lemmas.

Lemma 4.7. Assume that $\operatorname{asdim}\left(\operatorname{Co} Z_{1} \times \cdots \times \operatorname{Co} Z_{n}\right) \leq k$. Then for some $\bar{\varepsilon}>0$ there is a function $\delta:[0, \bar{\varepsilon}] \rightarrow[0,1]$ such that for every $\varepsilon \in(0, \bar{\varepsilon}), \tau \in(0,1)$ there exists a $(\delta(\varepsilon) \tau, \varepsilon \tau, k+1)$-covering of $Z_{1} \times \cdots \times Z_{n} \times[0, \tau]^{n}$ by open subsets of $Z_{1} \times \cdots \times Z_{n} \times \mathbb{R}^{n}$.

Proof. We put $Z=Z_{1} \times \cdots \times Z_{n}, X=\operatorname{Co} Z_{1} \times \cdots \times$ Co $Z_{n}$; let $h: Z \times \mathbb{R}^{n} \rightarrow X$ be the product of canonical projections. By assumption, for every $L>0$ there exists an $(L, M, k+1)$-covering $\mathcal{U}$ of $X$ with some $M<\infty$. We fix such a covering for $L>8 \delta_{\mathrm{H}^{2}}$, together with $\varepsilon \in(0, \bar{\varepsilon})$ and $\tau \in(0,1)$, and put $r=-\ln (\varepsilon \tau)+5 M / 2+c$, where $c=$ $c_{0}+2 \delta_{\mathrm{H}^{2}}$ is the constant defined before Claim 1 .

The annulus $\operatorname{An}_{[r, r+M / \varepsilon]} \subset X$ that consists of all $\left(x_{1}, \ldots, x_{n}\right) \in X$ with $r \leq\left|x_{i} o\right| \leq$ $r+M / \varepsilon, i=1, \ldots, n$, is covered by $\mathcal{U}(\varepsilon, \tau)=\left\{U \in \mathcal{U}: U \cap \operatorname{An}_{[r, r+M / \varepsilon]} \neq \varnothing\right\}$. We lift this covering to the covering $\mathcal{U}^{\prime}(\varepsilon, \tau)=h^{-1}(\mathcal{U}(\varepsilon, \tau))$ of $Z \times[r, r+M / \varepsilon]^{n}$ (the latter will be identified with $\left.Z \times[0, M / \varepsilon]^{n}\right)$.

We have $\operatorname{mesh}(\mathcal{U}(\varepsilon, \tau)) \leq M$. Applying Claim 4(2) with $l=M, R_{2}=r-M$ (note that $R_{2} \geq l$ ), and $R \geq R_{2}$, we obtain $\operatorname{mesh}^{\times} \mathcal{U}^{\prime}(\varepsilon, \tau) \leq(\varepsilon \tau, M)$, due to our choices. Applying Claim 4(1) with $R_{1}=r+M / \varepsilon, l=L$, and $R \leq R_{1}$, we obtain

$$
L^{\times} \mathcal{U}^{\prime}(\varepsilon, \tau) \geq\left(e^{-(r+M / \varepsilon)-L / 4-c}, L / 2\right)=\left(e^{-M / \varepsilon-L / 4-5 M / 2-2 c} \varepsilon \tau, L / 2\right),
$$

the last identity being ensured by the choice of $r$.

Consider the homothety "along the second factor", $Z \times[0, M / \varepsilon]^{n} \rightarrow Z \times[0, \tau]^{n}$, with coefficient $\varepsilon \tau / M$, and the image $\mathcal{V}$ of $\mathcal{U}^{\prime}$ under this homothety. Then $\operatorname{mesh}^{\times}(\mathcal{V}) \leq$ $(\varepsilon \tau, \varepsilon \tau), L^{\times}(\mathcal{V}) \geq\left(\delta_{0}(\varepsilon) \tau, \varepsilon \tau L /(2 M)\right)$ for $\delta_{0}(\varepsilon)=c_{1} \varepsilon e^{-M / \varepsilon}, c_{1}=e^{-L / 4-5 M / 2-2 c}$, and we obtain a covering with the required properties taking $\delta(\varepsilon)=\min \left\{\delta_{0}(\varepsilon), L \varepsilon /(2 M)\right\}$.

Lemma 4.8. Let $Z$ be a metric space. Suppose that for some $\bar{\varepsilon}>0$ there exists a function $\delta:[0, \bar{\varepsilon}] \rightarrow[0,1]$ such that for every $\varepsilon \in(0, \bar{\varepsilon}), \tau \in(0,1)$ there is a $(\delta(\varepsilon) \tau, \varepsilon \tau, k+1)$ covering of $Z \times[0, \tau]^{n}$ by open subsets of $Z \times[0, \infty)^{n}$. Then $\ell-\operatorname{dim}\left(Z \times[0,1]^{n}\right) \leq k$.

Proof. It suffices to find, for sufficiently large $m \in \mathbb{N}$, a $(c / m, C / m, k+1)$-covering of $Z \times[0,1]^{n}$ with $c, C$ depending only on $Z$.

We represent

$$
Z \times[0,1]^{n}=\bigcup_{\left(l_{1}, \ldots, l_{n}\right) \in A} Z \times\left[\frac{l_{1}}{m}, \frac{l_{1}+1}{m}\right] \times \cdots \times\left[\frac{l_{n}}{m}, \frac{l_{n}+1}{m}\right],
$$

where $A=\{0, \ldots, m-1\}^{n}$. We define $i: A \rightarrow S=\left\{0, \ldots, 2^{n}-1\right\}$ by

$$
i\left(l_{1}, \ldots, l_{n}\right)=\left(l_{1} \bmod 2\right) 2^{0}+\cdots+\left(l_{n} \bmod 2\right) 2^{n-1} .
$$

Let $\varepsilon_{0}=\min \{1 / 4, \bar{\varepsilon}\}$, and let $\varepsilon_{i+1}=\delta\left(\varepsilon_{i}\right) / 2, i \in S$. We fix a $\left(\delta\left(\varepsilon_{i}\right) / m, \varepsilon_{i} / m, k+1\right)$ covering $\mathcal{U}_{\left(l_{1}, \ldots, l_{n}\right)}$ of $Z \times\left[\frac{l_{1}}{m}, \frac{l_{1}+1}{m}\right] \times \cdots \times\left[\frac{l_{n}}{m}, \frac{l_{n}+1}{m}\right]$, where $i=i\left(l_{1}, \ldots, l_{n}\right)$. For $s \in S$, we put

$$
\mathcal{U}_{s}=\bigcup_{\left(l_{1}, \ldots, l_{n}\right) \in i^{-1}(s)} \mathcal{U}_{\left(l_{1}, \ldots, l_{n}\right)} .
$$

Then mesh $\left(\mathcal{U}_{s+1}\right) \leq L\left(\mathcal{U}_{s}\right) / 2$. Proceeding by induction on $s \in S$ and applying Lemma 3.1, we obtain a $(c / m, C / m, k+1)$-covering of $Z \times[0,1]^{n}$ with $c=\delta\left(\varepsilon_{2^{n}-1}\right), C=\varepsilon_{0}$.

Proof of Proposition 4.6. This follows immediately from the two lemmas above. 
4.1. Proof of the main results. It is known that any visual hyperbolic space $X$ can be quasi-isometrically embedded in the hyperbolic cone over its boundary at infinity $\partial_{\infty} X$, and that for a geodesic hyperbolic space $Y$ the hyperbolic cone over its boundary at infinity can be quasi-isometrically embedded in the space itself (see, e.g., [BS3]).

It follows that in these cases,

$$
\begin{aligned}
& \operatorname{asdim} X \leq \operatorname{asdim} \operatorname{Co}\left(\partial_{\infty} X\right), \\
& \operatorname{asdim} Y \geq \operatorname{asdim} \operatorname{Co}\left(\partial_{\infty} Y\right),
\end{aligned}
$$

and similar estimates are true for products of such spaces.

Now, Theorem 1.1 follows immediately from Proposition 4.1, Theorem 1.3 from Proposition 4.6, and Theorem 1.5 from Proposition 4.5.

For the proof of Corollary 1.4, we note that any Gromov hyperbolic group is a visual hyperbolic space with a compact, locally self-similar boundary at infinity; see [BL]. By Proposition 3.9, for the spaces of the family $\left\{a \partial_{\infty} \Gamma_{1} \times b \partial_{\infty} \Gamma_{2}: a, b \geq 1\right\}$ we have $\ell$-dim $\leq$ $n$ uniformly, $n=\operatorname{dim}\left(\partial_{\infty} \Gamma_{1} \times \partial_{\infty} \Gamma_{2}\right)$, for any hyperbolic groups $\Gamma_{1}, \Gamma_{2}$. By Theorem 1.1,

$$
\begin{aligned}
\operatorname{asdim}\left(\Gamma_{1} \times \Gamma_{2}\right) & \leq \ell-\operatorname{dim}\left(\partial_{\infty} \Gamma_{1} \times \partial_{\infty} \Gamma_{2}\right)+2 \\
& =\operatorname{dim}\left(\partial_{\infty} \Gamma_{1} \times \partial_{\infty} \Gamma_{2}\right)+2,
\end{aligned}
$$

where the last identity follows from [BL] and the fact that $\partial_{\infty} \Gamma_{1} \times \partial_{\infty} \Gamma_{2}$ is a compact, locally self-similar space. The reverse inequality

$$
\operatorname{asdim}\left(\Gamma_{1} \times \Gamma_{2}\right) \geq \operatorname{dim}\left(\partial_{\infty} \Gamma_{1} \times \partial_{\infty} \Gamma_{2}\right)+2
$$

follows from Theorem 1.3 and the inequalities

$$
\ell-\operatorname{dim}(Z \times[0,1]) \geq \operatorname{dim}(Z \times[0,1])=\operatorname{dim} Z+1,
$$

which are valid for any metric space $Z$.

Corollary 1.6 follows from Theorem 1.3 and Theorem 1.5.

\section{§. Applications}

As applications of Corollary 1.4, we have:

1) Examples of a strict inequality in the product theorem for the asymptotic dimension in the class of hyperbolic groups. Namely, in [Dr1, Dr2] it was proved that for every prime $p$, there is a hyperbolic Coxeter group $\Gamma_{p}$ with a Pontryagin surface $\Pi_{p}$ as the boundary at infinity. Then, by Corollary 1.4,

$$
\begin{aligned}
\operatorname{asdim}\left(\Gamma_{p} \times \Gamma_{q}\right) & =\operatorname{dim}\left(\Pi_{p} \times \Pi_{q}\right)+2 \\
& <\operatorname{dim} \Pi_{p}+\operatorname{dim} \Pi_{q}+2 \\
& =\operatorname{asdim} \Gamma_{p}+\operatorname{asdim} \Gamma_{q}
\end{aligned}
$$

for prime $p \neq q$ (the second identity follows from the main result of [BL]).

2) Examples of a strict inequality in the product theorem for the hyperbolic dimension (the hyperbolic dimension is a quasi-isometry invariant of metric space introduced in [BS2]). Indeed, let $\Gamma_{p}$ and $\Gamma_{q}$ for $p \neq q$ be as in 1). Then

$$
\begin{aligned}
\operatorname{hypdim}\left(\Gamma_{p} \times \Gamma_{q}\right) & \leq \operatorname{asdim}\left(\Gamma_{p} \times \Gamma_{q}\right) \\
& <\operatorname{dim} \Pi_{p}+\operatorname{dim} \Pi_{q}+2 \\
& =\operatorname{hypdim}\left(\Gamma_{p}\right)+\operatorname{hypdim}\left(\Gamma_{q}\right)
\end{aligned}
$$

(the above identity also follows from the main result of [BL] and [BS2]).

3) The formula $\operatorname{asdim}(\Gamma \times \mathbb{R})=\operatorname{asdim}(\Gamma)+1$ for any hyperbolic group $\Gamma$.

It is known that for a visual, proper, geodesic hyperbolic space $X$ we have

$$
\operatorname{dim}\left(\partial_{\infty} X\right)+1 \leq \operatorname{asdim} X \leq \ell-\operatorname{dim}\left(\partial_{\infty} X\right)+1
$$


The lower estimate is simple; the ideas of the proof are contained, e.g., in [Gr, 1.E $\left.\mathrm{E}_{1}^{\prime}\right]$. The upper estimate was proved in [Bu1, Bu2]. Corollary 1.6 gives a better estimate from below, making it possible to show that the first inequality in $(*)$ may be strict. More precisely, we give an example of a hyperbolic space with asymptotic dimension arbitrarily greater than the topological dimension of its boundary at infinity. Let $Z=\{0\} \cup\{1 / m$ : $m \in \mathbb{N}\}$, and let $X=\operatorname{Co} Z^{k}$. It is known that $\ell-\operatorname{dim}\left(Z^{k} \times[0,1]\right)=k+1$; see $[\mathrm{BL}]$. Then $\operatorname{asdim} X=k+1$, while $\operatorname{dim}\left(\partial_{\infty} X\right)=0$. The natural conjecture is that for every compact metric space $Y$ we have $\ell-\operatorname{dim}(Y \times[0,1])=\ell-\operatorname{dim}(Y)+1$. If this is true, then both inequalities in $(*)$ become identities.

\section{ACKNOWLEDGMENT}

I would like to thank Sergei Buyalo for many useful remarks and attention to this paper. I am also pleased to express my gratitude to the Max-Planck Mathematical Institute and the University of Münster for support and excellent working conditions while writing the paper.

\section{REFERENCES}

[BD] G. Bell and A. Dranishnikov, On asymptotic dimension of groups, Algebr. Geom. Topol. 1 (2001), 57-71. MR1808331 (2001m:20062)

[BoS] M. Bonk and O. Schramm, Embeddings of Gromov hyperbolic spaces, Geom. Funct. Anal. 10 (2000), no. 2, 266-306. MR1771428 (2001g:53077)

[Bu1] S. V. Buyalo, Asymptotic dimension of a hyperbolic space and capacity dimension of its boundary at infinity, Algebra i Analiz 17 (2005), no. 2, 70-95; English transl., St. Petersburg Math. J. 17 (2006), no. 2, 267-283. MR2159584 (2006d:31009)

[Bu2] - Capacity dimension and embedding of hyperbolic spaces into a product of trees, Algebra $\mathrm{i}$ Analiz 17 (2005), no. 4, 42-58; English transl., St. Petersburg Math. J. 17 (2006), no. 4, 581-591. MR2173936 (2006e:31008)

[BS1] S. Buyalo and V. Schroeder, Embedding of hyperbolic spaces in the product of trees, Geom. Dedicata 113 (2005), 75-93. MR2171299 (2006f:53055)

[BS2] _ Hyperbolic dimension of metric spaces, arXive:math. GT/0404525; Algebra i Analiz 19 (2007), no. 1, 93-108; English transl. in St. Petersburg Math. J. 19 (2008), no. 1. MR2319511

[BS3] Elements of asymptotic geometry (in preparation).

[BL] S. Buyalo and N. Lebedeva, Dimensions of locally and asymptotically self-similar spaces, arXiv:math.GT/0509433; Algebra i Analiz 19 (2007), no. 1, 60-92; English transl. in St. Petersburg Math. J. 19 (2008), no. 1. MR2319510

[Dr1] A. Dranishnikov, On the virtual cohomological dimensions of Coxeter groups, Proc. Amer. Math. Soc. 125 (1997), 1885-1891. MR1422863 (98d:55001)

[Dr2] _ Boundaries of Coxeter groups and simplicial complexes with given links, J. Pure Appl. Algebra 137 (1999), 139-151. MR1684267(2000d:20069)

[Dr3] - Open problems in asymptotic dimension theory, Preprint, 2006.

[Dr4] Cohomological approach to asymptotic dimension, arXive:math.MG/0608215.

[Gr] M. Gromov, Asymptotic invariants of infinite groups, Geometric Group Theory, Vol. 2 (Sussex, 1991) (G. A. Niblo, M. A. Roller, eds.), London Math. Soc. Lecture Note Ser., vol. 182, Cambridge Univ. Press, Cambridge, 1993, pp. 1-295. MR1253544 (95m:20041)

St. Petersburg Branch, Steklov Mathematical Institute, Russian Academy of Sciences, Fontanka 27, St. Petersburg 191023, Russia

E-mail address: lebed@pdmi.ras.ru

Received 19/JUN/2007 